\title{
Stationary planetary wave propagation in Northern Hemisphere winter - climatological analysis of the refractive index
}

\author{
Q. Li $^{1}$, H.-F. Graf ${ }^{1,2}$, and M. A. Giorgetta ${ }^{1}$ \\ ${ }^{1}$ Max Planck Institute for Meteorology, Hamburg, Germany \\ ${ }^{2}$ Center for Atmospheric Science, University of Cambridge, UK \\ Received: 19 July 2006 - Published in Atmos. Chem. Phys. Discuss.: 25 September 2006 \\ Revised: 8 January 2007 - Accepted: 9 January 2007 - Published: 15 January 2007
}

\begin{abstract}
The probability density on a height-meridional plane of negative refractive index squared $f\left(n_{k}^{2}<0\right)$ is introduced as a new analysis tool to investigate the climatology of the propagation conditions of stationary planetary waves based on NCEP/NCAR reanalysis data for 44 Northern Hemisphere boreal winters (1958-2002). This analysis addresses the control of the atmospheric state on planetary wave propagation. It is found that not only the variability of atmospheric stability with altitudes, but also the variability with latitudes has significant influence on planetary wave propagation. Eliassen-Palm flux and divergence are also analyzed to investigate the eddy activities and forcing on zonal mean flow. Only the ultra-long planetary waves with zonal wave number 1, 2 and 3 are investigated. In Northern Hemisphere winter the atmosphere shows a large possibility for stationary planetary waves to propagate from the troposphere to the stratosphere. On the other hand, waves induce eddy momentum flux in the subtropical troposphere and eddy heat flux in the subpolar stratosphere. Waves also exert eddy momentum forcing on the mean flow in the troposphere and stratosphere at middle and high latitudes. A similar analysis is also performed for stratospheric strong and weak polar vortex regimes, respectively. Anomalies of stratospheric circulation affect planetary wave propagation and waves also play an important role in constructing and maintaining of interannual variations of stratospheric circulation.
\end{abstract}

\section{Introduction}

The propagation and temporal variation of stationary planetary waves in the atmosphere are fundamental problems in observational and theoretical studies of dynamical meteorology. Charney and Drazin (1961) first investigated the ver-

Correspondence to: $\mathrm{Q} . \mathrm{Li}$

(qian.li@zmaw.de) tical propagation of planetary waves based on linear wave theory by calculating the effective index of refraction, which depends on the distribution of the zonal mean wind and concluded that the vertical propagation of stationary waves only happens when the zonal winds are westerly and not too strong. Afterwards, Dickinson (1968) investigated in more detail the control of zonal mean wind on planetary waves. He found that the presence of a "polar wave guide", created by the westerly jet of the winter upper stratosphere, refracts the propagating planetary waves, while the "equatorial wave guide", formed by the zero wind line in the tropics, absorbs rather than refracts the planetary waves. At the same time, by supposing the planetary waves in the stratosphere are propagating from the troposphere, Matsuno (1970) simulated the observed vertical structure and horizontal pattern of stationary planetary waves in the winter stratosphere by using a quasi-geostrophic model. He found that the results agree well with observations. Schoeberl and Geller (1977) used several models to test the wave response and also found great sensitivity of the vertical structure and propagation of planetary waves to the mean wind structure. Lin (1982) confirmed the sensitivity of vertical wave propagation to the mean wind structure. He also found that the latitudinal location of the polar night jet has a significant influence on the wave propagation. Chen and Robinson (1992) found that the key parameters controlling the vertical wave propagation include not only the vertical shear of zonal winds but also the vertical gradient of buoyancy frequency. Limpasuvan and Hartmann (2000) concluded that a large positive vertical shear of zonal wind will impede the upward propagation of planetary wave. However, Hu and Tung (2002) indicated that rather than impeding, the large positive vertical shear of zonal wind tends to enhance wave propagation. The enhancing influence of large positive vertical shear of zonal wind on wave propagation will be also confirmed in this current paper. All these studies indicated the controlling nature of the distribution of zonal wind and wind shear on planetary wave propagation.

Published by Copernicus GmbH on behalf of the European Geosciences Union. 
On the other hand, based on the wave-mean flow interaction theorem (Charney and Drazin, 1961; Eliassen and Palm, 1961; Andrews, 1985), planetary waves have also strong influences on winds. Planetary waves, generated in the troposphere by topography and diabatic heating, propagate upward from the troposphere to the stratosphere when zonal mean winds are positive and not exceeding the critical Rossby velocity (Charney and Drazin, 1961). These waves can change the stratospheric circulation when they break and get absorbed. However, when the zonal mean winds exceed the critical Rossby velocity, the upward propagating planetary waves will be refracted downward and in meridional direction (Matsuno, 1970). These refracted waves may in turn influence the planetary wave pattern in the troposphere and could also induce changes in the zonal mean flow of the troposphere. Boville (1984), by applying a general circulation model, showed that (unrealistically) large changes in the zonal mean wind distribution of the upper troposphere and lower stratosphere could produce dramatic alterations in the mid-tropospheric wave field. The wave number dependent critical Rossby velocity determines that only ultra-long waves have the possibility to propagate into the stratosphere in mid- and high latitudes (Charney and Drazin, 1961; Matsuno, 1970).

After the theoretical study of Charney and Drazin (1961), the refractive index of stationary planetary waves was firstly introduced as a diagnostic tool by Matsuno (1970) and has frequently been applied by a number of researchers thereafter (Lin, 1982; Huang and Gambo, 1982; Hu and Tung, 2002; Mukougawa and Hirootka, 2004; etc.) to investigate planetary wave propagation. The effective index of refraction of vertically propagating planetary waves of a given zonal wave number at a specific latitude depends primarily on the vertical structure of the atmosphere (Charney and Drazin, 1961; Matsuno, 1970). An important parameter is the vertical and meridional distribution of the zonal mean zonal wind. Theoretically planetary waves tend to avoid regions where the zonal winds are easterly or are westerly but exceed a critical, wave number dependent velocity. They are attracted towards regions where the value of refractive index squared is maximum. This theory provides an important paradigm of our thinking about the dynamic forcing of the stratosphere by the troposphere and is a useful diagnostic for vertical and meridional planetary wave propagation. However, as it is shown from the equation of refractive index (Matsuno, 1970), even though it is highly simplified, the high degree of freedom, introduced by the number of parameters used to compute the refractive index, still sometimes gives unsatisfying results (e.g. Mukougawa and Hirooka, 2004). Another problem with the traditional analysis of refractive index is that it generally lacks the ability to illustrate the climatological state of the atmosphere for propagation of planetary waves due to the overlapping of positive and negative refractive index squared, which often makes a mean refractive index squared hard to interpret. In order to improve this, the sea- sonal frequency distribution of days with negative refractive index squared $\left(f\left(n_{k}^{2}<0\right)\right)$ is introduced in this paper. This allows estimating the probability that the atmospheric state permits propagation of planetary waves in specific periods at different latitudes and heights and avoids the overlapping problem. This new analysis may help to enhance our understanding about the influence of the zonal mean wind structure on planetary wave vertical propagation from the troposphere to the stratosphere in a climatological sense.

Another popular diagnostic to investigate the planetary wave propagation, is the so-called Eliassen-Palm flux and its divergence (Eliassen and Palm, 1961; Andrews et al., 1987). Contrasting to the refractive index, the Eliassen-Palm flux (E-P flux hereafter) is a measure of wave activity and its divergence shows the eddy forcing on the zonal mean flow (Andrews et al., 1987).

In this study, a climatological analysis was performed in Northern Hemisphere boreal winter. Recently it was suggested that there are two different circulation regimes in the Northern Hemisphere stratospheric interannual variability (Christiansen, 2003). In order to clarify the planetary wave - mean flow interaction, the same analysis was also performed for the two different circulation regimes: strong and weak stratospheric polar vortex regimes.

\section{Data and methodology}

In this paper we used daily mean reanalysis data of geopotential height, wind and temperature from the National Centre for Environmental Prediction - National Centre for Atmospheric Research (NCEP-NCAR) (Kalnay et al., 1996; Kistler et al., 2001) for the Northern Hemisphere boreal winter months December, January and February (DJF hearafter) from 1958 to 2002 . The dataset is global on a $2.5^{\circ} \times 2.5^{\circ}$ grid at 17 vertical pressure levels extending from 1000 to $10 \mathrm{hPa}$. In this paper, only between the troposphere and the stratosphere the propagation and refraction of the stationary planetary waves is investigated, therefore, the $10 \mathrm{hPa}$ top level of NCEP-NCAR reanalysis data is high enough for this study. The climatological mean is constructed by averaging over 44 winters (1958-2002).

In this study, we applied the refractive index of stationary planetary waves developed by Andrews et al. (1987) based on the form of Matsuno (1970), and furthermore extended by Hu and Tung (2002):

$n_{k}^{2}(y, z)=\frac{\bar{q}_{\phi}}{\bar{u}}-\left(\frac{k}{a \cos \phi}\right)^{2}-\left(\frac{f}{2 N H}\right)^{2}$

where

$\bar{q}_{\phi}=\frac{2 \Omega}{a} \cos \phi-\frac{1}{a^{2}}\left[\frac{(\bar{u} \cos \phi)_{\phi}}{\cos \phi}\right]_{\phi}-\frac{f^{2}}{\rho_{0}}\left(\rho_{0} \frac{\bar{u}_{z}}{N^{2}}\right)_{z}$

is the meridional gradient of the zonal mean potential vorticity $(\mathrm{PV}) . \rho_{0}=\rho_{s} \exp (-z / H)$ is the background air density. 
Here $k, N, H, f, a, \Omega$ and $\phi$ denote the zonal wave number (ZWN hereafter), buoyancy frequency, scale height, Coriolis parameter, Earth radius, Earth rotation frequency and latitude respectively. Expansion of the third term on the right-hand side of Eq. (2) yields

$$
-\frac{f^{2}}{\rho_{0}}\left(\rho_{0} \frac{\bar{u}_{z}}{N^{2}}\right)_{z}=\left(\frac{f^{2}}{H N^{2}}+\frac{f^{2}}{N^{4}} \frac{d N^{2}}{d z}\right) \bar{u}_{z}-\frac{f^{2}}{N^{2}} \bar{u}_{z z}
$$

It can be seen from the above equations that the refractive index squared could be affected not only by atmospheric zonal wind and wind shear, but also by quadratic vertical shear of zonal mean zonal wind and atmospheric stability.

As discussed by Matsuno (1970), it is expected that planetary waves of wave number $\mathrm{k}$ are able to propagate in regions where $n_{k}^{2}>0$ and are refracted from regions where $n_{k}^{2}<0$ and that the larger the $n_{k}^{2}$ in a region, the easier it is for planetary waves to propagate there.

Based on the Charney and Drazin theorem (1961), only the ultra-long waves can propagate vertically from the troposphere to the stratosphere since the increasing ZWNs correspond to decreasing critical Rossby velocities. Therefore in this paper, for single zonal wave number (ZWN) stationary planetary waves, only ZWN 1, 2 and $3(\mathrm{k}=1,2$ and 3 , respectively) planetary waves are investigated.

It can be concluded from Eq. (1) that the refractive index reflects the atmospheric state for propagation of stationary planetary waves. On the other hand, analysis of the EliassenPalm flux (E-P flux) and its divergence is applied in this study to investigate the stationary planetary wave activities and wave forcing on the mean flow in Northern Hemisphere winter (DJF). The quasi-geostrophic version of E-P flux in spherical geometry (Andrew et al., 1987) is employed:

$\vec{F}=\rho_{0}\left[0, \overline{\Phi_{\phi}^{\prime} \Phi_{\lambda}^{\prime}} / f^{2} a, \overline{\Phi_{z}^{\prime} \Phi_{\lambda}^{\prime}} / N^{2}\right]$

and its divergence is

$D_{F}=\frac{\nabla \times \vec{F}}{\rho_{0} a \cos \phi}$

where $\Phi$ is geopotential field.

By quasi-geostrophic approximation, Eq. (4) can be presented as:

$\vec{F}=\left[0,-\rho_{0}(\cos \phi) \overline{v^{\prime} u^{\prime}}, \rho_{0} a(\cos \phi) f \overline{v^{\prime} \theta^{\prime}} / \theta_{0 z}\right]$

From Eq. (6), it can be seen that, the meridional vector component of E-P flux indicates the eddy momentum flux and the vertical vector component represents the eddy heat flux, while the divergence of E-P flux (Eq. 5) implies the eddy forcing on the mean flow (Andrew et al., 1987; Hartmann and Lo, 1998; Chen et al., 2002).

\section{Definition of frequency of negative refractive index squared of stationary planetary waves}

As a powerful diagnostic to investigate the control of the atmosphere on the propagation of stationary planetary waves, the refractive index has been frequently applied since the first form introduced by Matsuno (1970). However, as mentioned above, the traditional analysis of refractive index lacks the ability to illustrate the climatology of the atmospheric state supportive of vertical propagation of planetary waves due to the overlapping of positive and negative value of $n_{k}^{2}$. Figure 1 shows the mean of $n_{k}^{2}$ of 44 boreal winters (1958-2002), single winter (1958-1959 and 1978-1979), and single days (1 January 1959 and 1 January 1979) for ZWN 1, 2 and 3 planetary waves, respectively. It can be seen for the ZWN 1 planetary wave, that there are differences between the means of $n_{1}^{2}$ for 44 winters (Fig. 1a) and for single winters (Fig. 1d) for the negative value of $n_{1}^{2}$ near the northern pole. Differences can also be found between the mean of 44 winters (Fig. 1a) and single day (Fig. 1j) not only at the pole but also in midlatitudes. On the other hand, the mean of $n_{1}^{2}$ is distributed differently for different single winters (Figs. 1d, g). Clear differences can also be observed between single days from different winters (Figs. 1j, m). Similar conclusions could be drawn for ZWN 2 and 3 waves.

In Fig. $1, n_{k}^{2}$ shows different patterns when the time mean is calculated. The calculation of the time mean of $n_{k}^{2}$ induces a reduction of information, which makes it is hard to interpret the results. In order to avoid the problem caused by overlapping of positive and negative $n_{k}^{2}$ when the time mean is of interest, the seasonal frequency distribution of days with negative $n_{k}^{2}\left(f\left(n_{k}^{2}<0\right)\right.$ hereafter $)$ is introduced in this section.

The $f\left(n_{k}^{2}<0\right)$ is produced from the daily mean data by counting the number of days with negative $n_{k}^{2}$ in the whole dataset at all latitudes and heights. Dividing by the total number of days, a two dimensional probability distribution of refraction of planetary waves is obtained. At places where the probability of negative $n_{k}^{2}$ is small, planetary waves have a good chance to propagate, while large probabilities indicate that very seldom a planetary wave is allowed to propagate across these places. This analysis does not provide any indication of the direction and total energy flux of planetary waves, but it turns out that it clearly provides a picture of probable wave propagation by "channels" of low probability of negative $n_{k}^{2}$.

It should be indicated that without the overlapping problem the climatology of E-P flux and divergence is constructed by 44 winters averaging. 
(a)
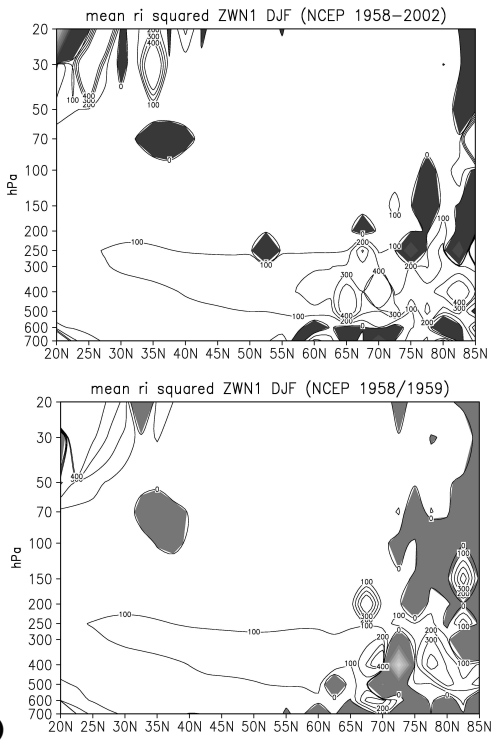

(d)

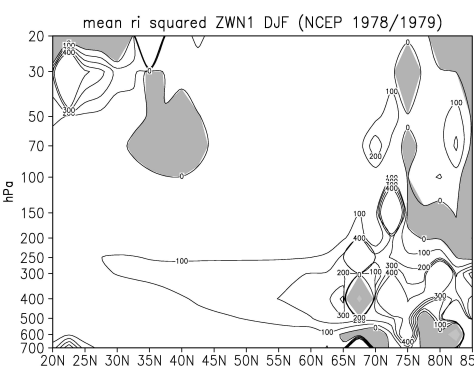

(g)

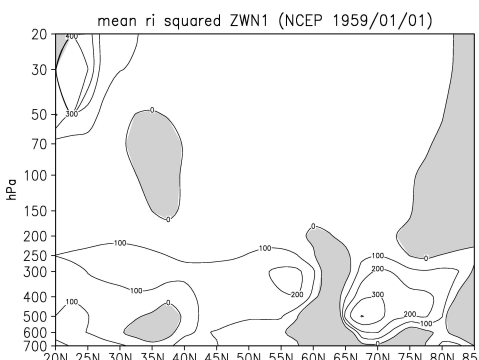

(j)

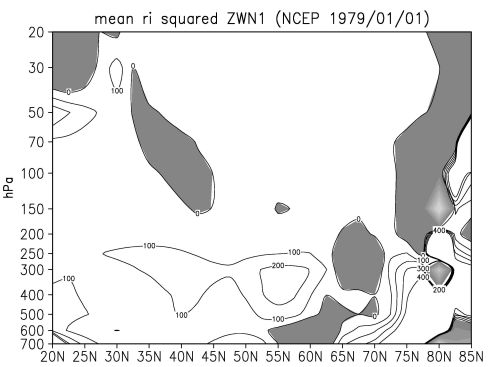

(b)
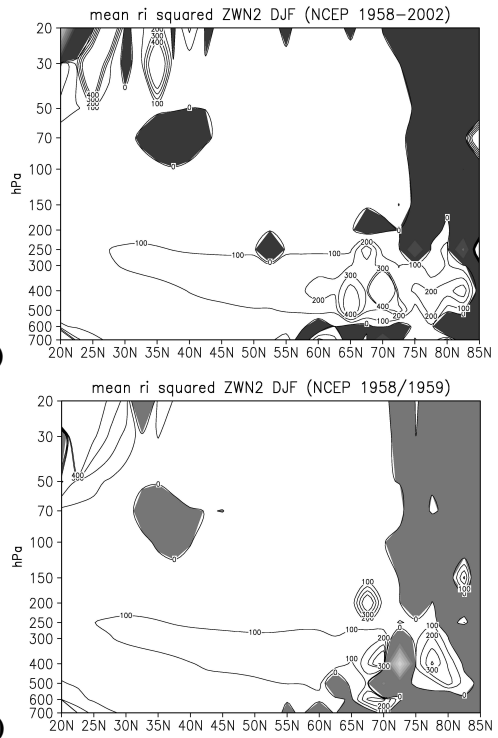

(e)

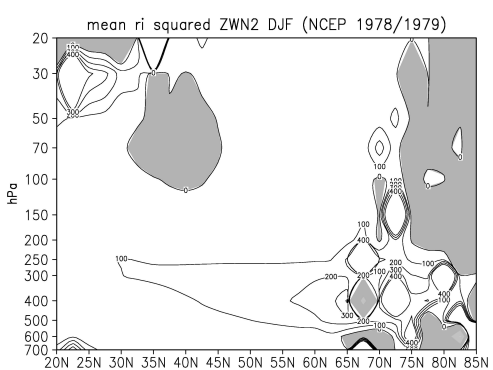

(h)

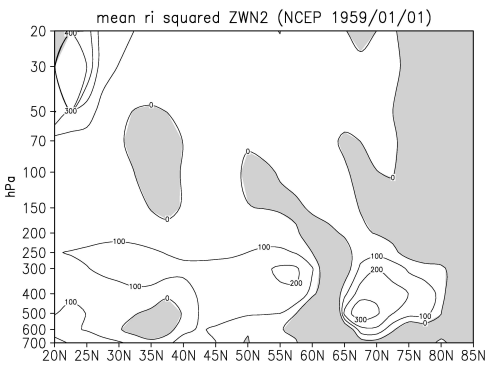

k)

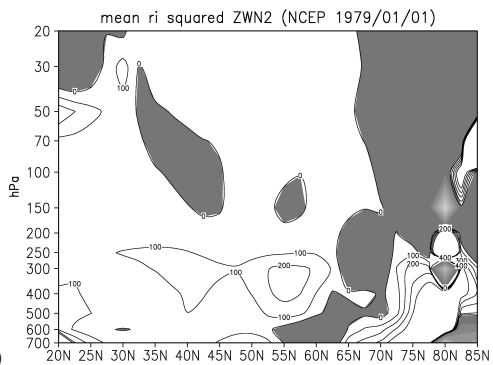

(c)
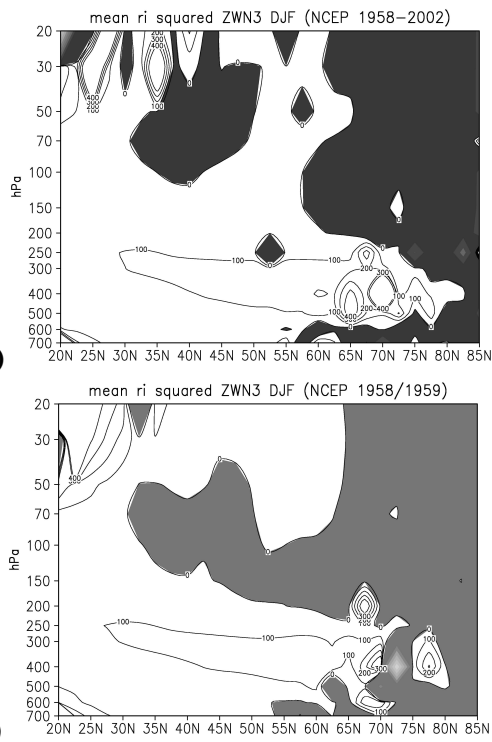

(f)

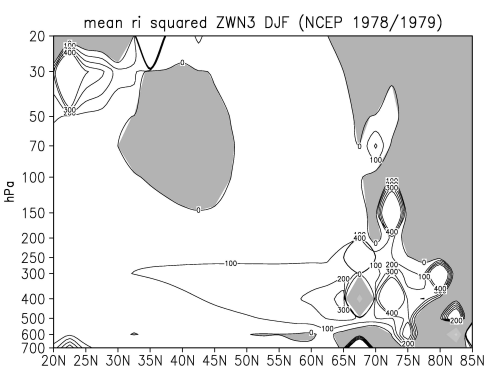

(i)

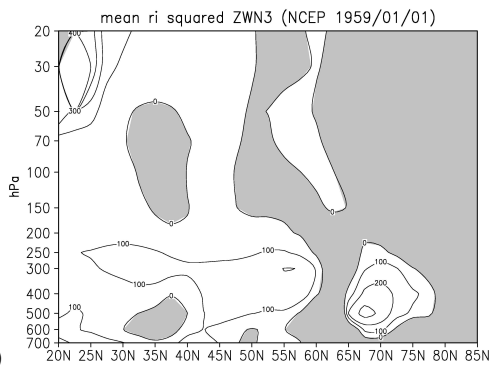

(o)

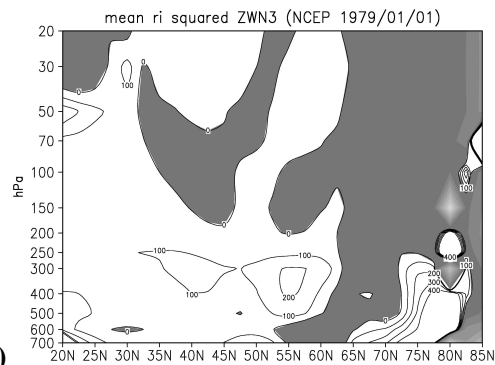

Fig. 1. Refractive index squared $\left(a^{2} n^{2}\right)$ for ZWN 1 (first column) ZWN 2 (second column) and ZWN 3 (third column) stationary waves (NCEP/NCAR RA, 1958-2002). Mean of $a^{2} n^{2}$ of 44 boreal winters (December, January and February) (first row), mean of 1958-1959 DJF (second row), mean of 1978-1979 DJF (third row), 1 January 1959 (fourth row) and 1 January 1979 (last row). In the plot, contours range from 0 to 400 with an interval of 100 , regions with negative values are shaded. 


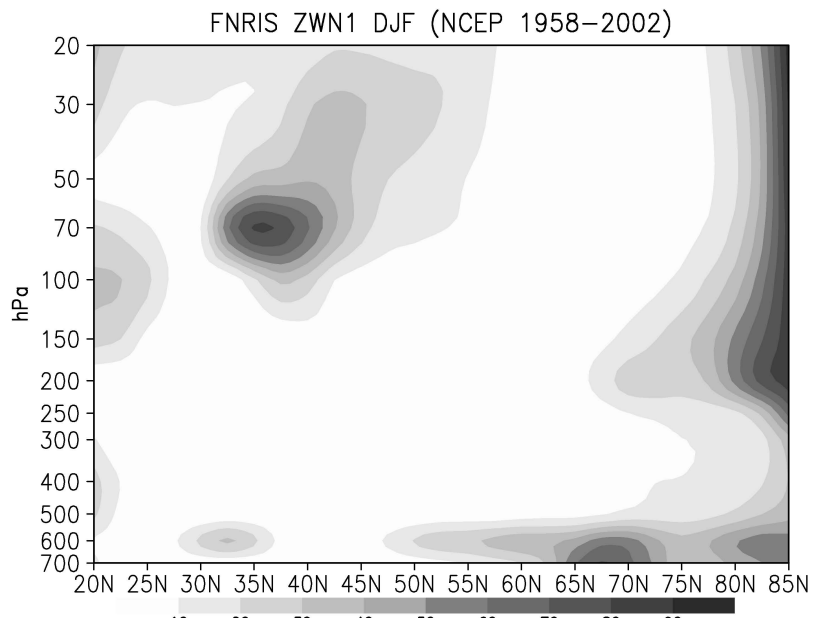

(a)
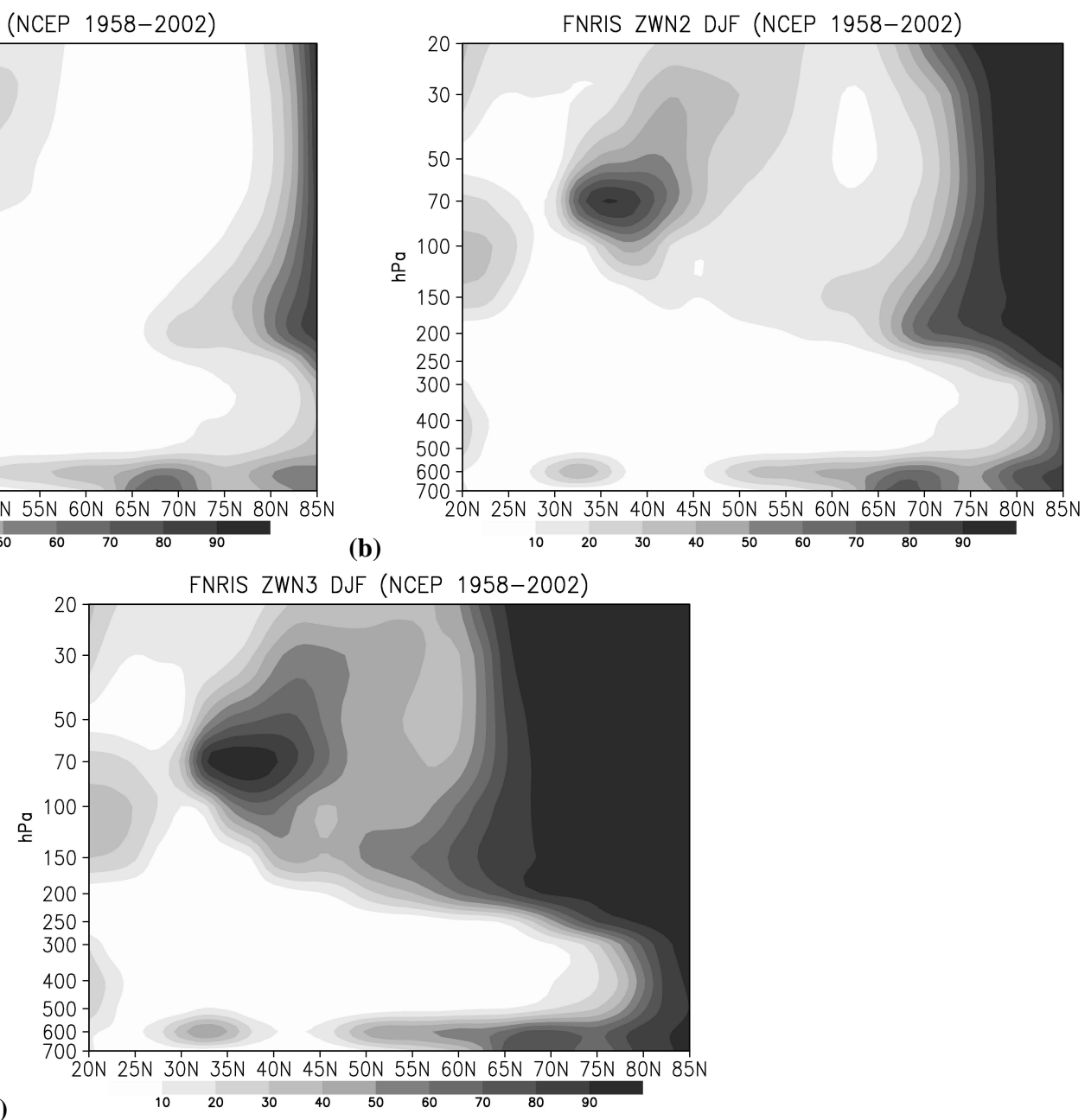

(b)

Fig. 2. $f\left(n_{k}^{2}<0\right)$ for stationary planetary waves in DJF (NCEP/NCAR RA 1958-2002); (a) ZWN 1 wave; (b) ZWN 2 wave; (c) ZWN 3 wave. Unit of $f\left(n_{k}^{2}<0\right)$ is percent.

\section{Results and discussions}

\subsection{Boreal winter}

Figure 2 shows the distribution of $f\left(n_{k}^{2}<0\right)$ for stationary planetary waves in Northern Hemisphere boreal winter (DJF) of 1958-2002 on the meridional plane for ZWN 1, 2 and 3 waves, respectively. Since this analysis was performed based on the quasi-geostrophic theory, which is not appropriate in the tropics, all figures were drawn only north of $20^{\circ} \mathrm{N}$. In order to enhance the clearness of the atmospheric state for planetary wave propagation in both troposphere and stratosphere and to avoid boundary effects of data sets, patterns are shown only between $700 \mathrm{hPa}$ and $20 \mathrm{hPa}$ in figures.

There are common characteristics for all ZWN 1, 2 and 3 waves. It is clear that the planetary waves have rather a big chance to propagate upward in the middle and upper troposphere for all ZWN 1, 2 and 3 (Figs. 2a, b and c). This cor- responds to the general knowledge that the ultra-long waves can propagate from the troposphere vertically to the stratosphere (Charney and Drazin, 1961; Matsuno, 1970). It can be observed that the waves exited from the lower troposphere (below $500 \mathrm{hPa}$ ) have less probability to propagate upward at higher latitudes (north of $50^{\circ} \mathrm{N}$ ). On the other hand, Fig. 2 shows that the potential of vertical propagation from the troposphere to the stratosphere is not given on large parts of the meridional plane. This differs from former results of the general distribution of mean $n_{k}^{2}$, which showed positive values of $n_{k}^{2}$ for ZWN 1 on the whole meridional plane (see Fig. 1a; Huang and Gambo, 1982, Fig. 7; Limpasuvan and Hartmann, 2000, Fig. 8). It is noted that there is a small area above the subtropical tropopause where the value of $f\left(n_{k}^{2}<0\right)$ is rather high for all ZWN 1, 2 and 3 waves. This small area was also found in $\mathrm{Hu}$ and Tung (2002, Figs. 5c and d) for ZWN 1 wave. The location of this small area can be related 

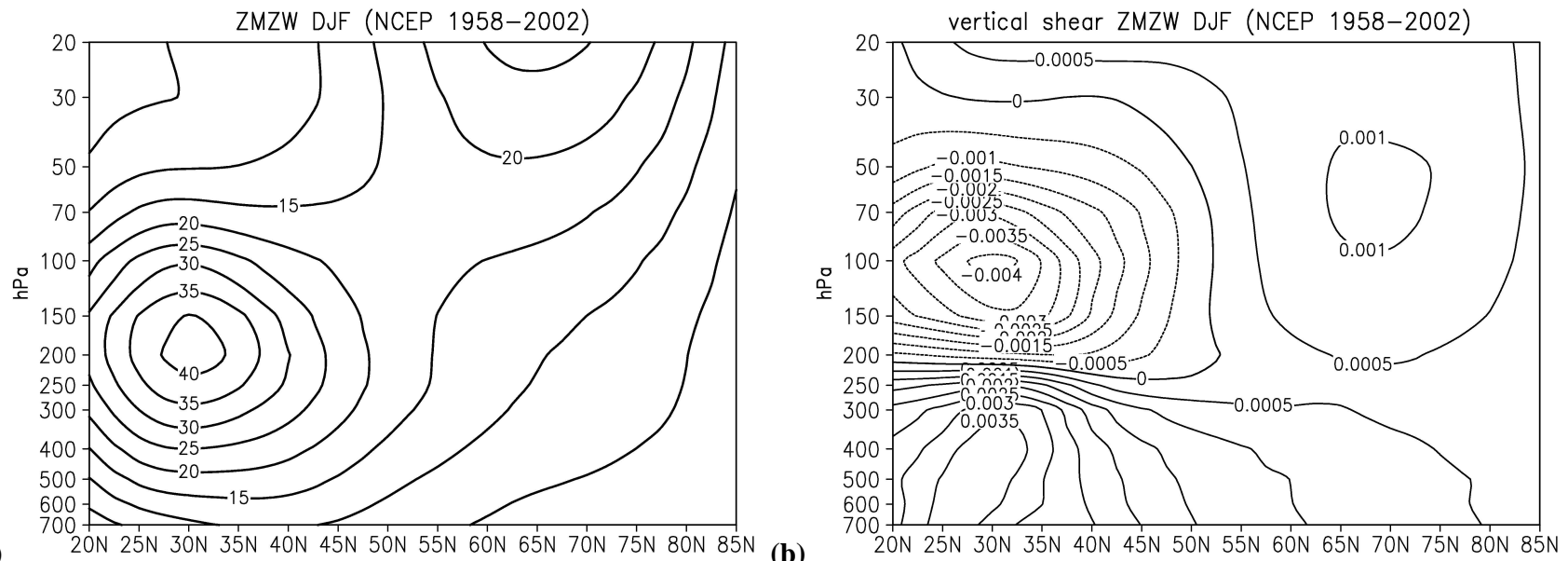

(a)

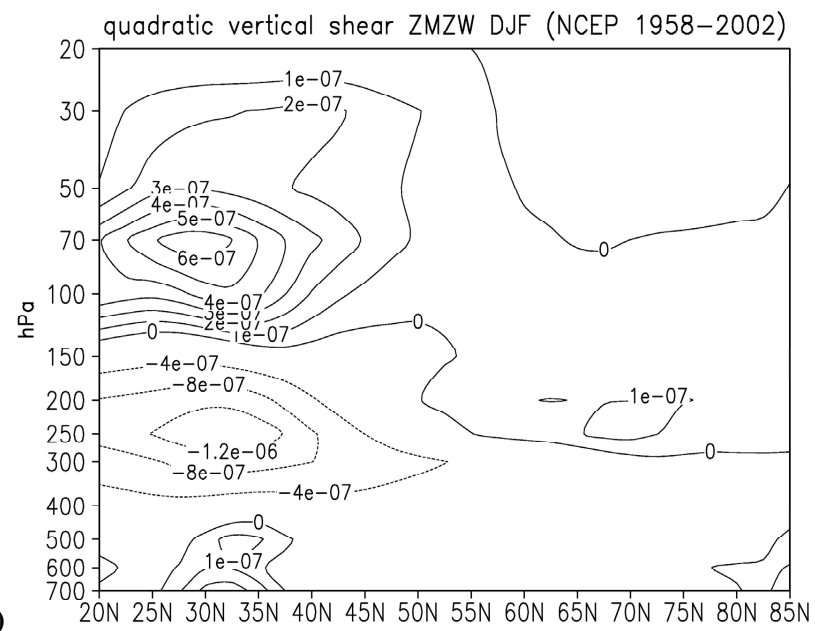

Fig. 3. Zonal mean zonal wind $\bar{u}(\mathrm{~m} / \mathrm{s})$ (a), vertical shear of zonal mean zonal wind $\frac{\partial \bar{u}}{\partial z}\left(\mathrm{~s}^{-1}\right)(\mathbf{b})$, and quadratic vertical shear of zonal mean zonal wind $\frac{\partial^{2} \bar{u}}{\partial z^{2}}\left(\mathrm{~m}^{-1} \mathrm{~s}^{-1}\right)$ (c) on meridional plane in DJF (NCEP/NCAR RA 1958-2002).

to another small area above the subtropical tropopause where the negative vertical shear of the zonal mean zonal wind is centered (Fig. 3b). This finding corresponds to Chen and Robinson's (1992) conclusion, that larger negative vertical wind shear impedes wave propagation across the tropopause, while the distribution of quadratic wind shear $\frac{\partial^{2} \bar{u}}{\partial z^{2}}$ (Fig. 3c) on the meridional plane does not show any clear connection with the impeded wave propagation across the subtropical tropopause. This point has been also indicated by $\mathrm{Hu}$ and Tung (2002). Another common characteristic is the existence of a "channel" from the troposphere to the stratosphere at mid-latitudes between high values of $f\left(n_{k}^{2}<0\right)$ at rather higher latitudes and the small area indicated above. The presence of this "channel" corresponds to the "wave guide" indicated by Huang and Gambo (1982, Fig. 7) from the lower troposphere at $40^{\circ} \mathrm{N}$ toward high latitudes stratosphere. Hence. our analysis is able to cover all the individual findings that were made previously by other authors and this convinces us of the appropriateness of our method.
There are also obvious differences between ZWN 1, 2 and 3 waves of the distribution of $f\left(n_{k}^{2}<0\right)$. First of all, comparing Figs. 2a, b and c representing $f\left(n_{k}^{2}<0\right)$ for ZWN 1, 2 and 3 , respectively, the value of $f\left(n_{k}^{2}<0\right)$ on the whole meridional plane increases with increased ZWNs, which implies that the probability of wave propagation decreases with the increasing ZWNs. This obviously agrees well with the ZWN-depending critical Rossby velocity (Charney and Drazin, 1961). The small area above the subtropical tropopause with rather higher values of $f\left(n_{k}^{2}<0\right)$ strengthens with increasing ZWNs, but the general location does not move. The width of the narrow column at the north pole with higher $f\left(n_{k}^{2}<0\right)$ for ZWN 1 wave (Fig. 2a) increases strongly equatorward with increasing ZWNs (Figs. 2b andc). These two enhanced patterns finally narrow the "channel" indicated above for wave propagation from the troposphere to the stratosphere. This mainly reduces the probability of propagation of ZWN 3 and smaller waves from the troposphere to the stratosphere. 
It should be mentioned that in the calculation of the refractive index the buoyancy frequency squared $N^{2}$ is treated as a variable of both altitude and latitude $\left(N^{2}(\phi, z)\right)$. The buoyancy frequency represents the stability of atmosphere. In some other studies, the buoyancy frequency was treated as a constant (Matsuno, 1970; Huang and Gambo, 1982; Andrews et al., 1987), or as a variable of altitudes only (Chen and Robinson, 1992; Limpasuvan and Harmann, 2000; Hu and Tung, 2002). Chen and Robinson (1992) found that the variation of $N^{2}$ is not as negligible as it has been hypothesized in other applications (Matsuno, 1970; Andrews et al., 1987). They concluded that the propagation of planetary waves from the troposphere to the stratosphere is sensitive to the distribution of $N^{2}(z)$ especially near the subtropical tropopause. The value of $N^{2}$ near the subtropical and extratropical tropopause has fairly strong vertical and meridional gradients. This can also be concluded from the comparison of $N^{2}(z)$ and $N^{2}(y, z)$ (Fig. 4). These gradients of $N^{2}$ influence the distribution of planetary wave propagation. Comparing $f\left(n_{k}^{2}<0\right)$ which considers the $N^{2}$ as being variable of only altitudes (not shown) with the results considering the $N^{2}$ as variable of both latitudes and altitudes (Fig. 2), it was found that the vertical gradient near the extratropical tropopause has an effect on the probability of planetary wave propagation for all ZWN 1, 2 and 3 waves at the same region. We conclude that the strong vertical gradient of $N^{2}$ reduces the probability of wave propagation. This is consistent with the results of Chen and Robinson (1992). It can also be concluded that the vertical distribution of $N^{2}$ plays a more important role for propagation of planetary waves than previously suggested (Andrew et al., 1987; Chen and Robinson, 1992). However, the vertical distribution of $N^{2}$ changes significantly with changing latitudes, especially $300 \mathrm{hPa}$ to $70 \mathrm{hPa}$ (Fig. 4). As it was observed in the analysis of $f\left(n_{k}^{2}<0\right)$, the planetary waves can propagate from the troposphere to the stratosphere in the "channel" crossing the tropopause. Therefore the great change of vertical distribution of $N^{2}$ around tropopause should not be neglected. It is necessary to treat the buoyancy frequency $N^{2}(y, z)$ and not to use the simplified $N^{2}(z)$.

As discussed above, the analysis of $f\left(n_{k}^{2}<0\right)$ only gives the information of the state of the zonal atmospheric mean flow for the probability of propagation of stationary planetary waves. The refractive index implies that it is useful in determining the influence of the mean flow on the waves. On the other hand, the Eliassen-Palm (E-P) flux is of fundamental importance in determining the wave forcing on the zonal mean flow. To study the wave activities on the meridional plane, E-P flux will help to understand the dynamics of planetary waves - mean flow interaction between the troposphere and the stratosphere. Therefore, E-P flux for planetary waves is also applied to study the climatology of planetary wave propagation in this paper.

Figure 5 shows the cross section of E-P flux and its divergence on the meridional plane in Northern Hemisphere win-

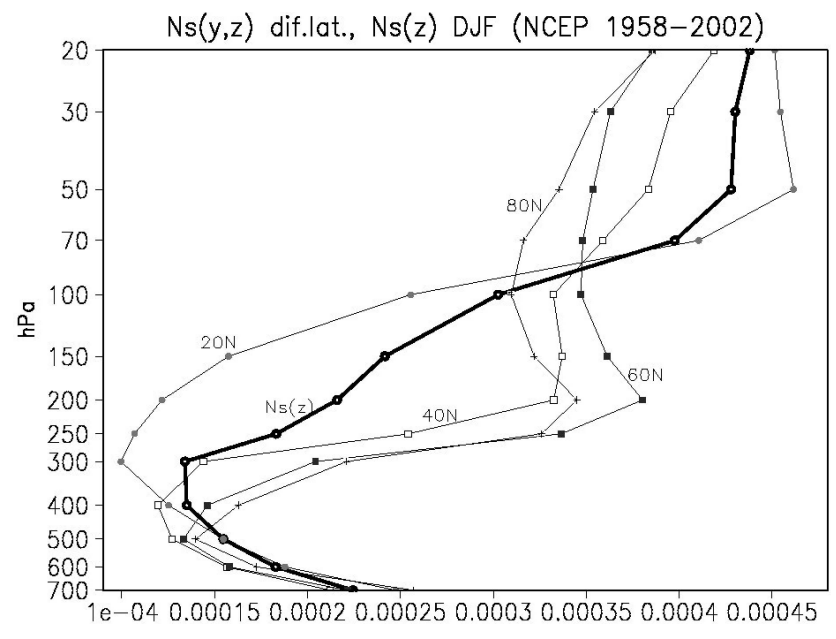

Fig. 4. Buoyancy frequency of Northern Hemisphere winter (DJF) $N^{2}(z)$ profile (dark line) and $N^{2}(y, z)$ profiles on different latitudes. Unit of $N^{2}$ is s $\mathrm{s}^{-2}$.

ter (DJF) for ZWN 1, 2, 3, ZWN 1+2+3 and all ZWNs waves, respectively. It should be noted that, as shown in Fig. 5, the difference between ZWN $1+2+3$ and all ZWNs (Fig. 5f) is negligible comparing with ZWN 1, 2 and 3 (Figs. 5a, b, and c) which indicates that the shorter planetary waves with bigger ZWNs (>3) are responsible for much less (more than one order of magnitude) eddy activity and eddy forcing on the zonal mean flow. This clearly corresponds to the Charney and Drazin Theorem (1961). Therefore, as mentioned before, in this study only ZWN 1, 2 and 3 waves are investigated, $\mathrm{ZWN} 1+2+3$ waves are supposed to represent the all ZWNs waves.

There are two major branches of the vectors of E-P flux for all ZWN's stationary waves and they are exerted from the extratropical troposphere. One branch of the vectors starts from the lower troposphere and points upward across the tropopause to the lower and middle stratosphere (Fig. 5d). It is shown by Eq. (6) that the meridional and vertical vectors of E-P flux represent the eddy momentum flux and eddy heat flux, respectively (Andrew et al., 1987; Hartmann and Lo, 1998; Chen et al., 2002). Therefore, it can be concluded that in Northern Hemisphere winter, the eddy heat flux dominates the wave activities between the troposphere and the lower and mid- stratosphere, and therefore baroclinic energy prevails there. These upward vectors of E-P flux also correspond with the "channel" shown by $f\left(n_{k}^{2}<0\right)$ (Fig. 2). Another branch of vectors starts also from the surface but turns equatorward in the upper troposphere. This implies the importance of the eddy momentum flux in the subtropical upper troposphere. There are also two major regions where the E-P flux converges, which indicates an easterly zonal momentum forcing exerted by planetary waves on the atmosphere. One is located in the extratropical upper troposphere and another in the subpolar mid- stratosphere. The latter one is relatively weaker. 
EP flux and div. ZWN1 stat. DJF (NCEP 1958-2002)

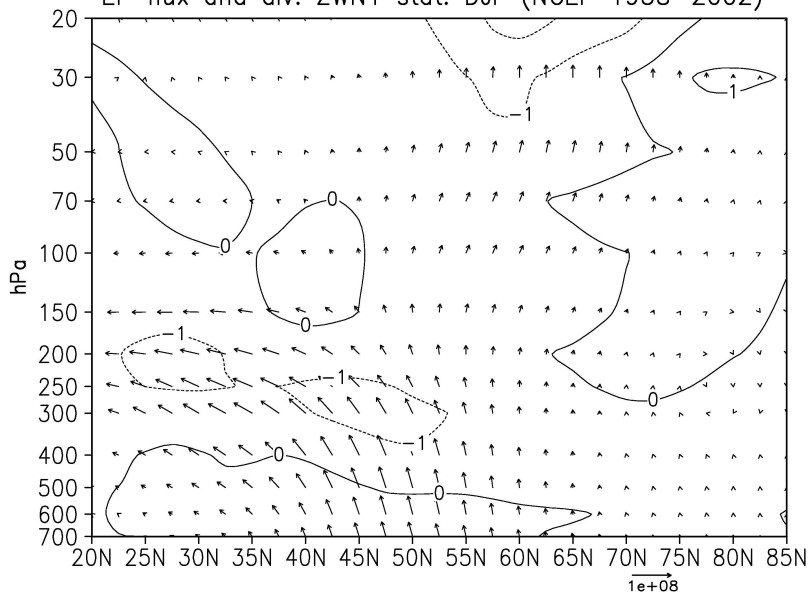

(a)

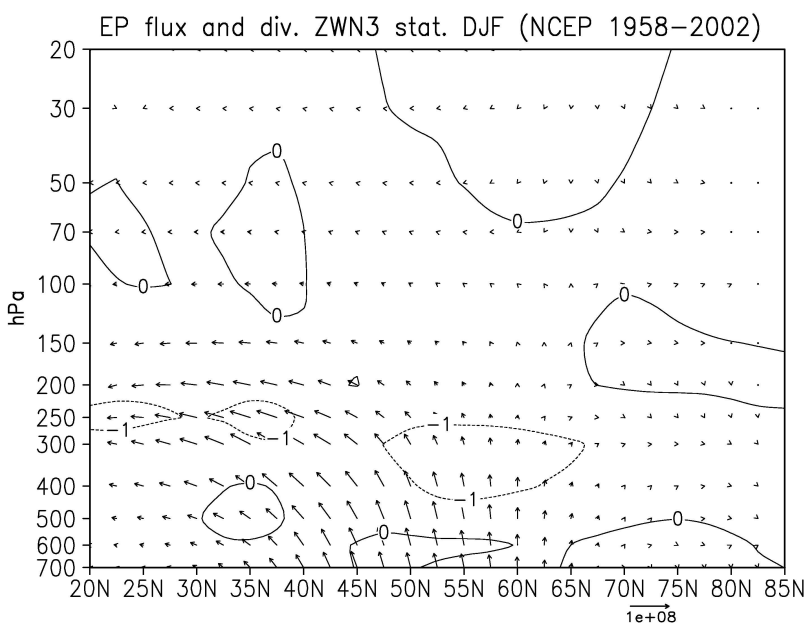

(c)

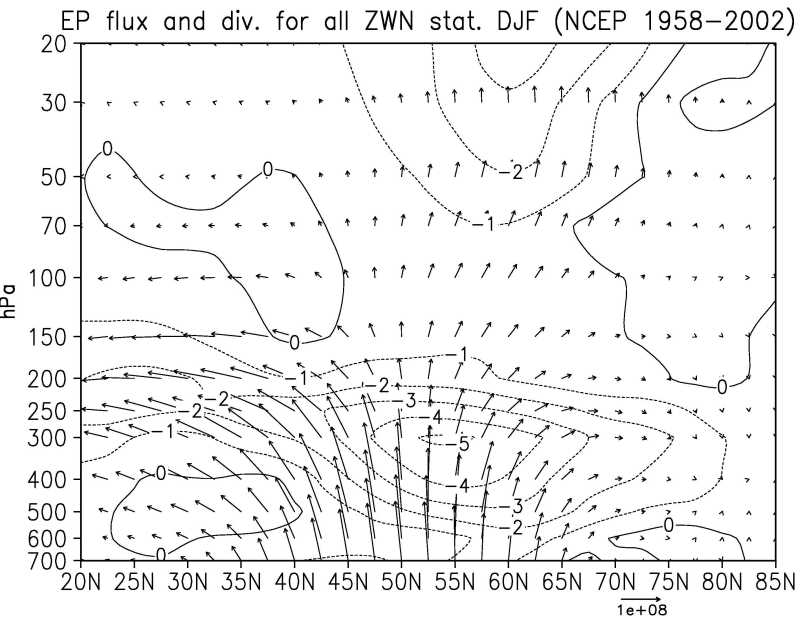

(b)
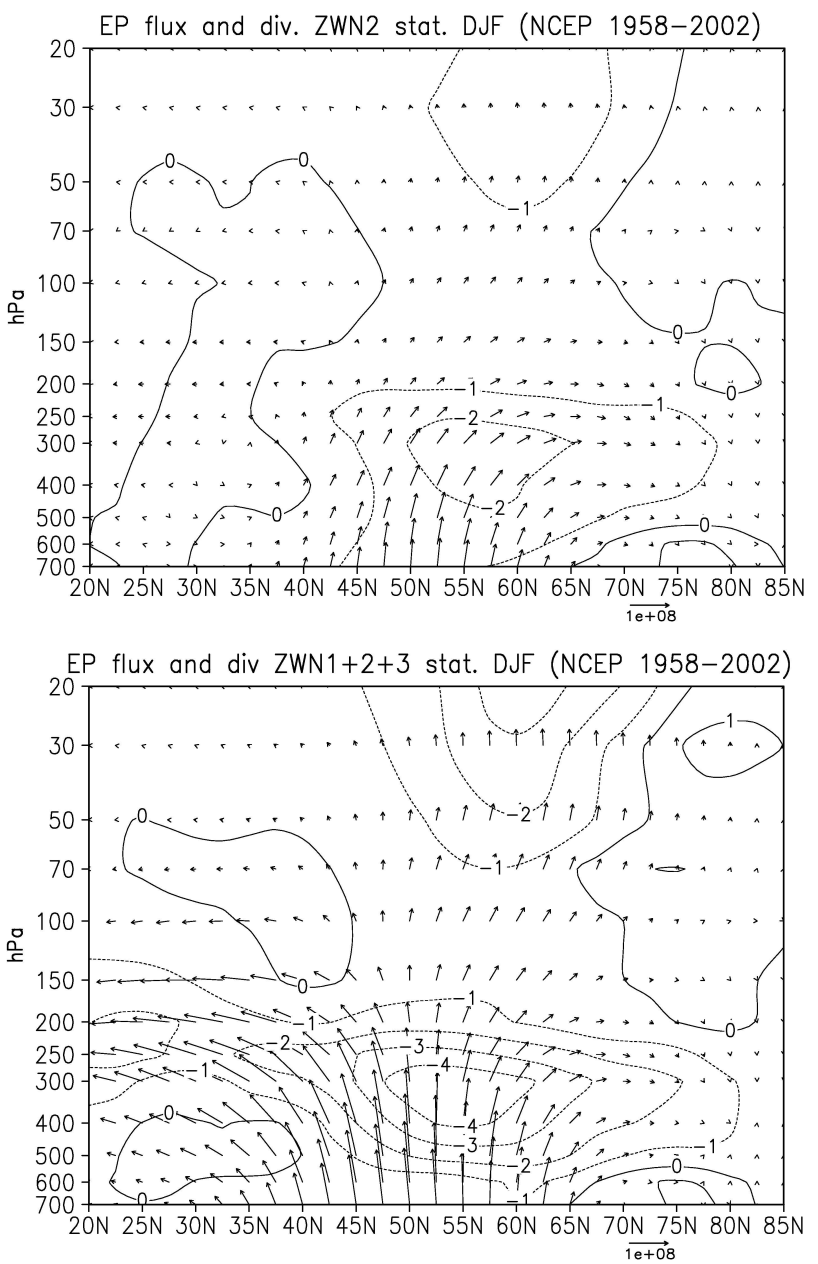

(d)

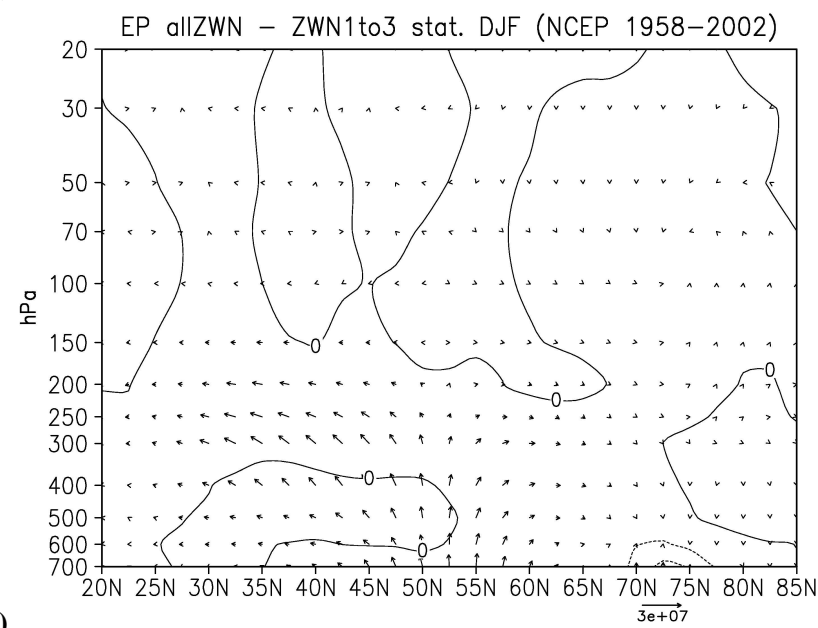

Fig. 5. Cross section of E-P flux for stationary planetary waves in DJF (NCEP/NCAR RA 1958-2002); (a) ZWN 1 wave; (b) ZWN 2 wave; (c) ZWN 3 wave; (d) ZWN 1+2+3 waves; (e) all ZWN waves; (f) difference between all ZWN waves and ZWN 1+2+3 waves. Divergence contour interval is $1 \mathrm{~ms}^{-1}$ day $^{-1}$, the unit of vector is $\mathrm{kg} \mathrm{s}^{-2}$. 
Considering the contribution of different ZWN waves, E$P$ flux and divergence of ZWN 1 wave (Fig. 5a) show the same pattern as in the all ZWN waves (Fig. 5e) with two major branches of vectors and two major convergent areas, while the amplitudes are relatively smaller. However, there is only one major branch of vectors observed for both ZWN 2 wave and ZWN 3 wave. In ZWN 2 wave (Fig. 5b) only the upward branch exists and the amplitudes of the vectors become weaker especially in the subpolar lower and midstratosphere compared with ZWN 1 wave (Fig. 5a). In contrast, for ZWN 3 wave (Fig. 5c) only the equatorward branch in the troposphere still exists. Above the two major E-P flux convergent regions, similar as for ZWN 1 waves, they are reduced in ZWN 2 waves (Fig. 5b). However the convergence in the subpolar stratosphere disappears in ZWN 3 waves (Fig. 5c) even though there is potential possibility for wave propagation (Fig. 2c).

To briefly summarize, as discussed above, stationary planetary waves can transfer eddy heat energy from the troposphere to the stratosphere and eddy momentum energy to the subtropical upper troposphere by the two major branches of E-P flux vectors. They also excert easterly zonal momentum forcing on the atmosphere at the extratropical upper troposphere and at the subpolar lower and mid- stratosphere by the two E-P flux convergent regions. The ZWN 1 wave contributes most for wave propagation from the troposphere to the stratosphere, compared with ZWN 2 and 3 waves.

\subsection{Strong and weak stratospheric polar vortex regimes}

In the study of planetary wave propagation, the wave-mean flow interaction should be considered with caution since the distribution of zonal mean zonal wind (Charney and Drazin, 1961; Matsuno, 1970) and vertical shear of zonal wind (Lin, 1982; Chen and Robinson, 1992; Hu and Tung, 2002) plays a key role on the planetary wave propagation in both the troposphere and the stratosphere. The stratospheric circulation is characterized by strong westerly flow in subpolar latitudes (Fig. 3a) in Northern Hemisphere winter. This socalled stratospheric polar vortex usually forms in early winter as a consequence of the cooling of the northern polar region due to the strong cooling above the winter pole. It is well known that this stratospheric polar vortex undergoes intraseasonal and interannual anomalies (Baldwin and Dunkerton, 1999, 2001; Castanheira and Graf, 2003; Christiansen, 2003: Limpasuvan et al., 2005; Perlwitz and Graf, 1995, 2000, 2001a, b) which generally can be observed by the zonal wind distribution and are separated into strong and weak polar vortex regimes. In the Northern Hemisphere, the linkage between stratosphere and troposphere appears most obvious when the stratospheric polar vortex undergoes unusually strong variation in wind strength and temperature (Limpasuvan et al., 2005).

Christiansen (2003) presented evidence of the existence of two different circulation regimes in the Northern Hemisphere stratospheric interannual variability. The regimes correspond to strong stratospheric polar vortex and weak stratospheric polar vortex. By studying the PDFs of the leading PC of the geopotential height over the Northern Hemisphere averaged over the winter half-year (from October to March), he found the existence of a bimodal probability density distribution in middle and lower Northern stratosphere. Similar results are found, if averages are taken over three winter months (December, January and February) (Christiansen, 2003). However, if monthly means are used, Christiansen (2003) found that the PDFs are unimodal as Gillet et al. (2002) reported for daily values of temperature data, which probably lack the ability to illustrate the biomodal regimes. As indicated by Palmer (1993), the presence of higher frequencies can obscure the existence of bimodality.

By studying the zonal mean zonal wind at $50 \mathrm{hPa}$ and $65^{\circ} \mathrm{N}$, Walter $(2003,2004)$ found that the strength of stratospheric polar vortex shows 30-days persistence. They counted all months in which the monthly mean zonal wind at $50 \mathrm{hPa}$ and $65^{\circ} \mathrm{N}$ is stronger than $20 \mathrm{~m} / \mathrm{s}$ to the strong polar vortex regime and those months in which the monthly mean zonal wind at $50 \mathrm{hPa}$ and $65^{\circ} \mathrm{N}$ is weaker than $10 \mathrm{~m} / \mathrm{s}$ were counted to the weak vortex regime. This threshold has been discussed in Castanheira and Graf (2003) but based on daily mean data. They concluded that this regime threshold is somehow arbitrary for the real atmosphere but it reflects the critical Rossby velocity $(20 \mathrm{~m} / \mathrm{s})$ for ZWN 1 planetary wave near the polar circle for a climatological Northern Hemisphere zonal wind profile.

In this study, in order to increase the clearness of the results and to avoid the unimodal problem when the monthly mean is used, the definition of strong and weak polar vortex regimes is based on daily means, which was also applied in Castanheira and Graf (2003). Considering the daily means of zonal mean zonal wind at $50 \mathrm{hPa}$ and $65^{\circ} \mathrm{N}\left(\bar{u}_{50}\left(65^{\circ} \mathrm{N}\right)\right)$ of Northern Hemisphere winter (November to March), the strong polar vortex regime (SVR hereafter) consists of all consecutive days in which $\bar{u}_{50}\left(65^{\circ} \mathrm{N}\right)>20 \mathrm{~m} / \mathrm{s}$ for at least 30 days. The weak polar vortex regime (WVR hereafter) is defined as the regime including all consecutive days in which $0<\bar{u}_{50}\left(65^{\circ} \mathrm{N}\right)<10 \mathrm{~m} / \mathrm{s}$ and also is lasting at least 30 days. Based on this, the data are subdivided into the two polar vortex regimes. The strong polar vortex regime (SVR) and weak polar vortex regime (WVR) therefore will be discussed separately below.

Table 1 shows the periods of SVR (Table 1a) and WVR (Table 1b) in all winter seasons of 1958 to 2002 based on the zonal mean zonal wind data from NCEP-NCAR reanalysis daily mean data. There are 11 SVR cases and 12 WVR cases. Including all days, SVR covers 777 days and WVR 529 days. The shortest period of SVR includes 45 (22 January 1959 to 7 March 1959, Table 1a) days, which is longer than the one of WVR with 33 days (26 February 1981 to 30 March 1981, Table 1b). This corresponds to the general knowledge that the stratospheric circulation in Northern Hemisphere winter 

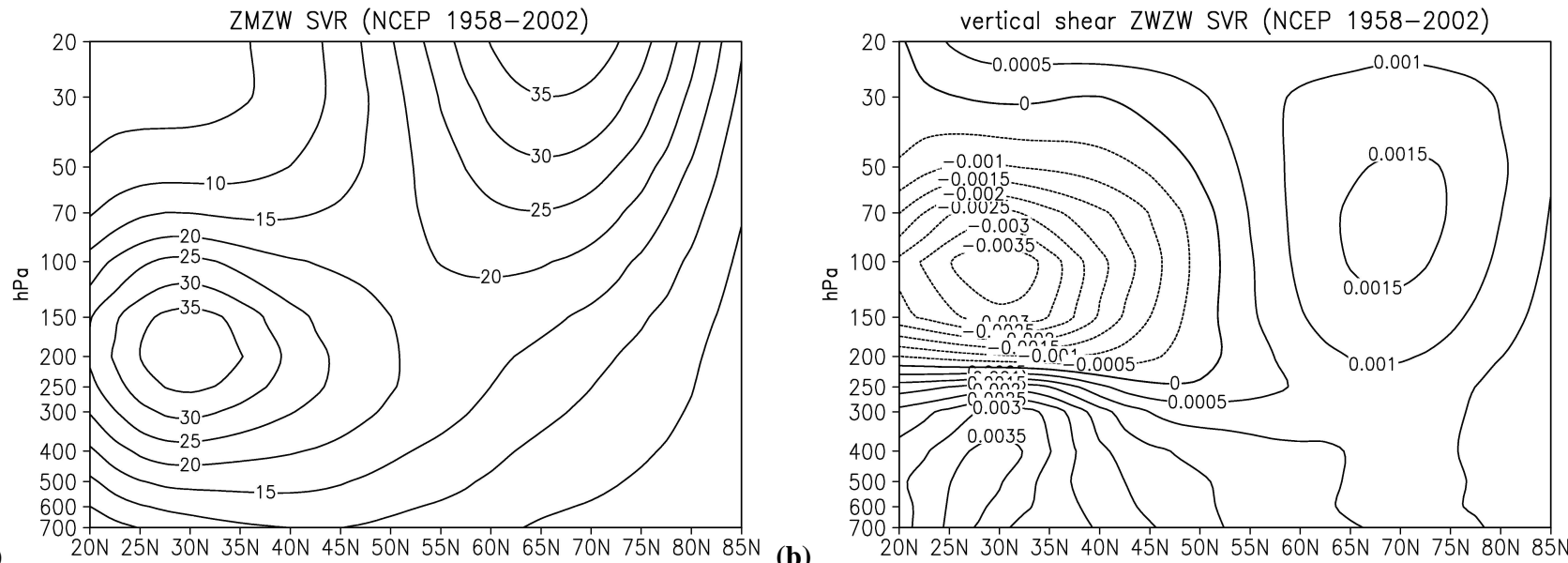

(a)

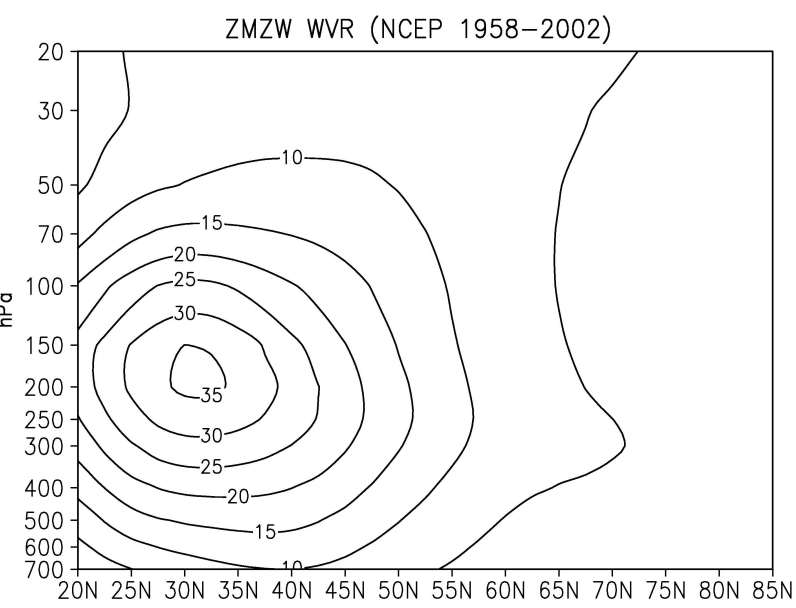

(b)

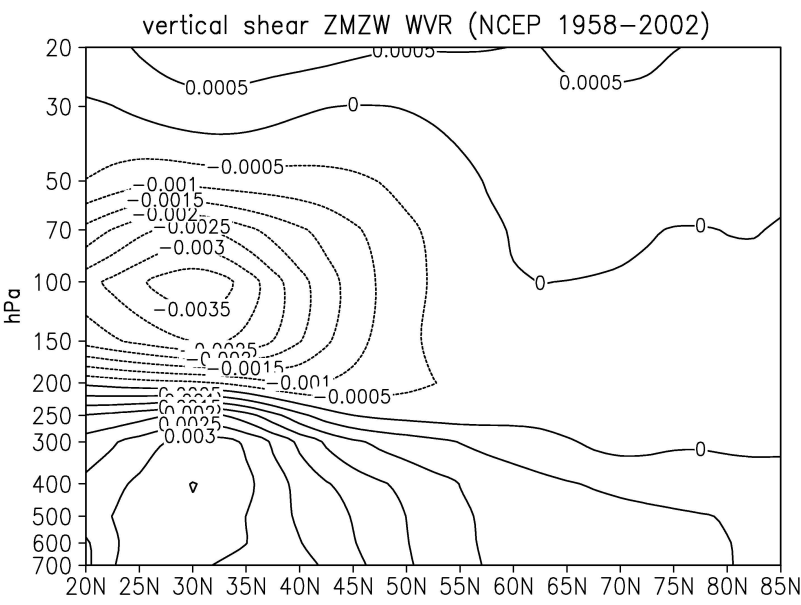

(c)

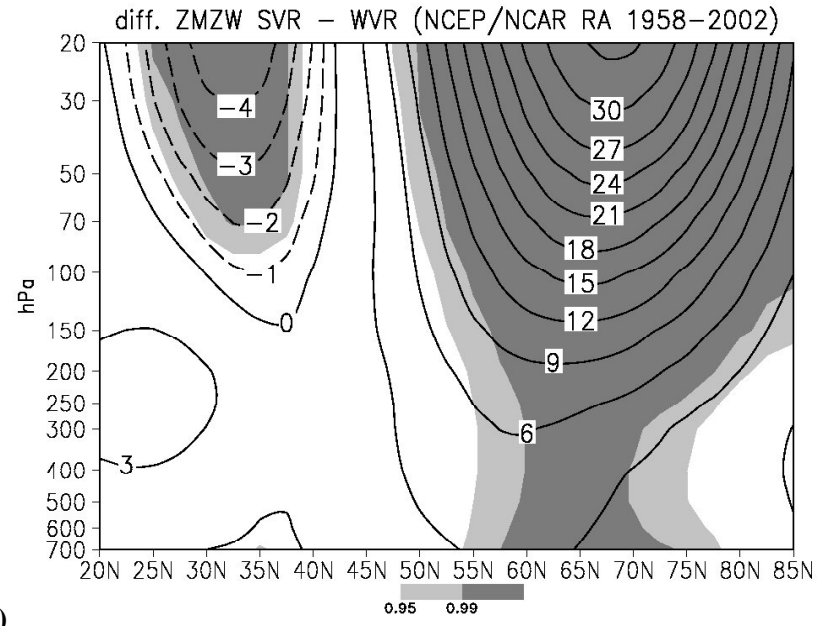

(e)

(d)

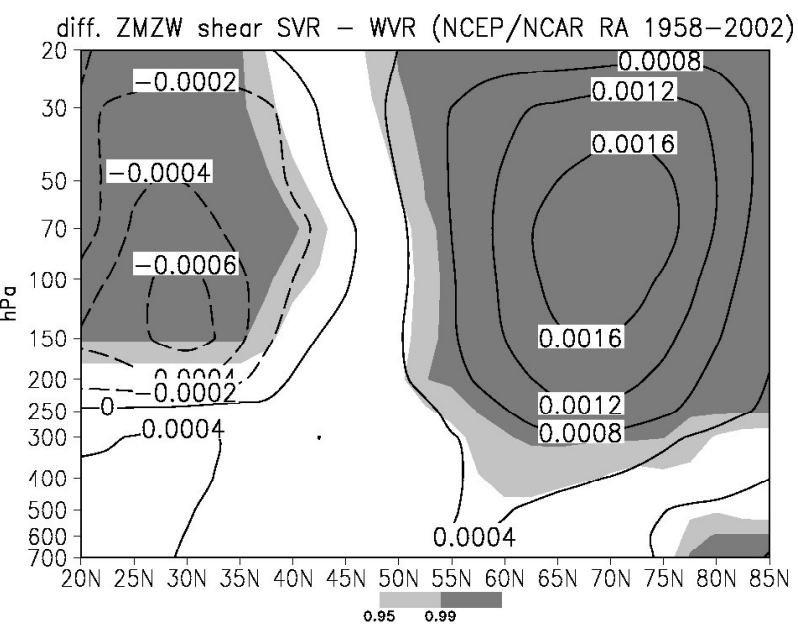

Fig. 6. Zonal mean zonal wind ( $\mathrm{m} / \mathrm{s}$ ) (left column) and its vertical shear $\left(\mathrm{s}^{-1}\right)$ (right column) on meridional plane for SVR (first row), WVR (second row) and difference between SVR and WVR (bottom row) (NCEP-NCAR RA 1958-2002). The area with significant level more than $95 \%$ is mediumly shaded, with significant level more than $99 \%$ is heavily shaded.

prefers to stay stronger westerly. It is obvious that there is no one winter in which both SVR and WVR happened and this corresponds to the understanding that the anomalies of strato- spheric polar vortex in Northern Hemisphere winter should be considered as annual patterns (Baldwin and Dunkerton, 1999, 2001; Limpasuvan and Hartmann, 2000). 
The distribution of zonal mean zonal wind and vertical shear for SVR and WVR is shown in Fig. 6, respectively. Both zonal mean zonal wind (Fig. 6a) and wind shear (Fig. 6b) of SVR are similar with patterns in all DJF (Figs. 2a, b) except that the strength of wind at subpolar latitudes is much stronger in SVR. It should be indicated that there exists a positive wind shear at the subpolar latitudes in the lower stratosphere for both DJF (Fig. 2b) and SVR (Fig. 6b), but the value is bigger in SVR. On the other hand, both the strong westerlies and the positive wind shear at the subpolar stratosphere are reduced in WVR (Figs. 6c, d). In order to clarify the mean flow anomalies, the difference of zonal mean zonal wind and wind shear between SVR and WVR is drawn in Figs. 6e and $\mathrm{f}$.

The $f\left(n_{k}^{2}<0\right)$ is also analyzed in SVR and WVR for ZWN 1, 2 and 3 stationary planetary waves, respectively (Fig. 7). Comparing with DJF, similar general patterns exist in SVR and WVR except for the variation of the "channel" at mid latitudes between the troposphere and the stratosphere with increased ZWNs. For ZWN 1 wave, the "channel" which represents the high probability of wave propagation is narrower in SVR (Fig. 7a) than in WVR (Fig. 7b), and in DJF (Fig. 2a). This means that the ZWN 1 stationary planetary wave has a smaller chance to force the stratospheric circulation in SVR than in WVR (Castanheira and Graf, 2003) and in DJF. On the other hand, the probability of wave propagation between troposphere and stratosphere in WVR is bigger. This point has been addressed by several other studies (Perlwitz and Graf, 2001a; Castanheira and Graf, 2003; Walter, 2003). This can also be observed for ZWN 2 wave (Figs. 7c, d). For ZWN 3 wave, on the other hand, the "channel" in which a wave has a chance to propagate from the troposphere to the stratosphere is almost totally "blocked" in SVR (Fig. 6e). This is different from DJF (Fig. 2c) and WVR (Fig. 7f). Based on the analysis of $f\left(n_{k}^{2}<0\right)$, it can be concluded that the anomalies of atmospheric mean flow influence the probability of stationary planetary wave propagation.

In order to enhance our understanding of wave-mean flow interaction, the E-P flux and divergence are also analyzed in SVR and WVR. Figure 8 shows the cross section on the meridional plane for E-P flux and divergence in SVR and WVR, for ZWN 1, 2, 3 and 1+2+3 waves, respectively.

The major patterns of E-P flux and divergence as shown in all DJF (Fig. 5) are well conserved in both SVR and WVR (Fig. 8). For ZWN 1+2+3, in both SVR and WVR, there exist the two major branches of vectors, as well as the two major convergent areas. However, there is also an obvious difference between the two stratospheric polar vortex regimes. The convergent area at the subpolar stratosphere is strengthened both in amplitude and area in WVR (Fig. 8h). In fact, almost the whole extratropical and subpolar stratosphere is covered by the convergence of E-P flux in WVR, which means the wave-exerted easterly zonal force the on mean flow is dominating in the stratosphere. A similar variation in WVR is also
Table 1. Lasting periods of polar vortex regimes; (a) strong polar vortex regime (SVR) and (b) weak polar vortex regime (WVR) (NCEP/NCAR RA 1958 to 2002).

(a)

\begin{tabular}{cc}
\hline Starting dates & Ending dates \\
\hline 26 Jan 1958 & 31 March 1958 \\
1 Nov 1960 & 27 Dec 1960 \\
28 Feb 1963 & 13 March 1963 \\
12 Nov 1965 & 25 Dec 1965 \\
20 Dec 1968 & 27 Jan 1969 \\
26 Feb 1981 & 30 March 1981 \\
28 Dec 1984 & 13 Feb 1985 \\
24 Nov 1987 & 28 Dec 1987 \\
14 Nov 1996 & 22 Dec 1996 \\
9 Dec 1998 & 11 Jan 1999 \\
8 Nov 2000 & 22 Dec 2000 \\
4 Feb 2001 & 31 March 2001 \\
\hline
\end{tabular}

(b)

\begin{tabular}{cc}
\hline Starting dates & Ending dates \\
\hline 22 Jan 1959 & 7 March 1959 \\
24 Dec 1963 & 28 Feb 1964 \\
3 Jan 1967 & 31 March 1967 \\
1 Dec 1975 & 24 March 1976 \\
20 Nov 1987 & 14 Jan 1987 \\
16 Dec 1988 & 17 Feb 1989 \\
17 Dec 1989 & 31 March 1990 \\
2 Nov 1990 & 29 Dec 1990 \\
24 Nov 1991 & 18 Jan 1992 \\
5 Dec 1992 & 11 Feb 1993 \\
26 Nov 1994 & 18 Jan 1995 \\
\hline
\end{tabular}

shown for ZWN 1 and 2 waves, but with less intensity. Oppositely, there is intensified divergence at polar stratosphere in SVR, which can induce westerly zonal force on the mean flow at the polar lower and mid-stratosphere. Limpasuvan and Hartmann (2000) also indicated that the baroclinic contribution can lead to strong westerly forcing in the middle stratosphere at high latitudes.

In order to clarify the potential influence of wave activities on mean flow anomalies, the differences of E-P flux and divergence between in SVR and WVR are shown in Fig. 9. The equatorward vectors and the divergence of E$\mathrm{P}$ flux in the extratropical upper troposphere are stronger in SVR, while the upward vectors and divergence at the polar lower and mid-stratosphere are stronger in WVR (Fig. 8h). Therefore, since the meridional vectors of E-P flux represent the eddy momentum flux and the vertical vectors represent the eddy heat flux (Andrew et al., 1987; Chen et al., 2002), we can conclude that eddy momentum flux in the subtropical 
(a)

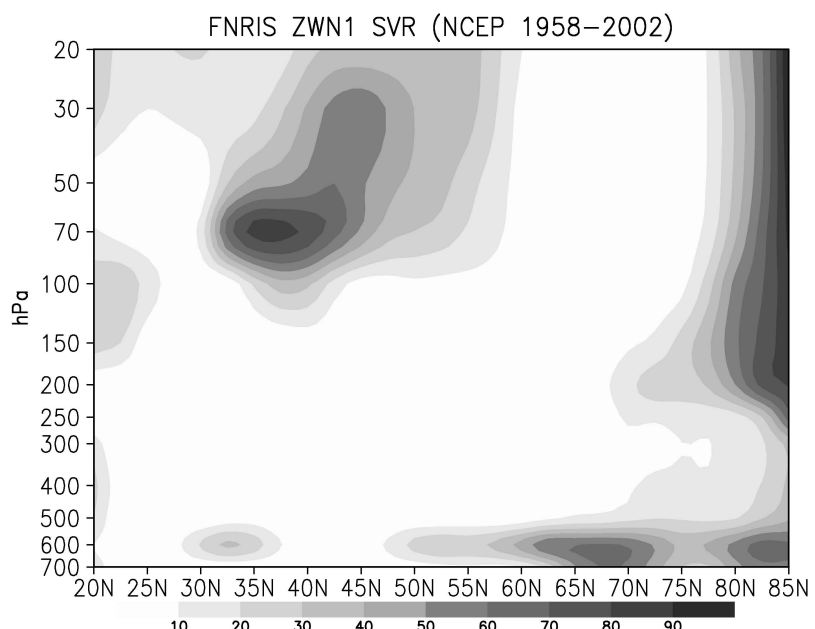

FNRIS ZWN2 SVR (NCEP 1958-2002)

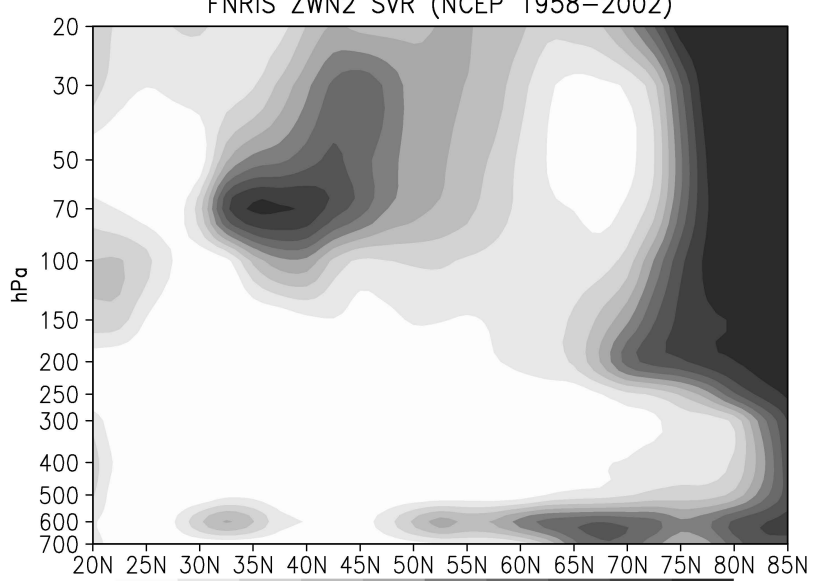

(c)

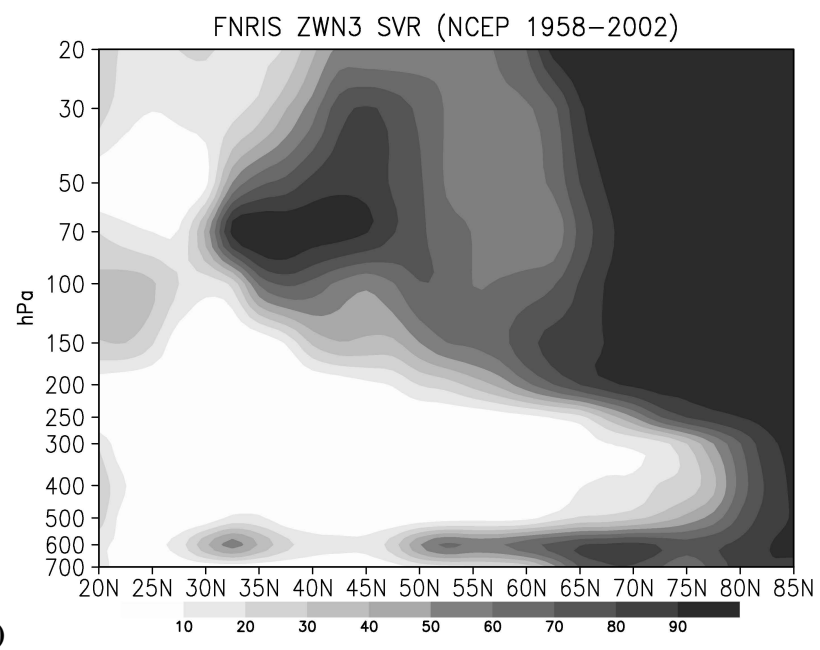

(e) (f)

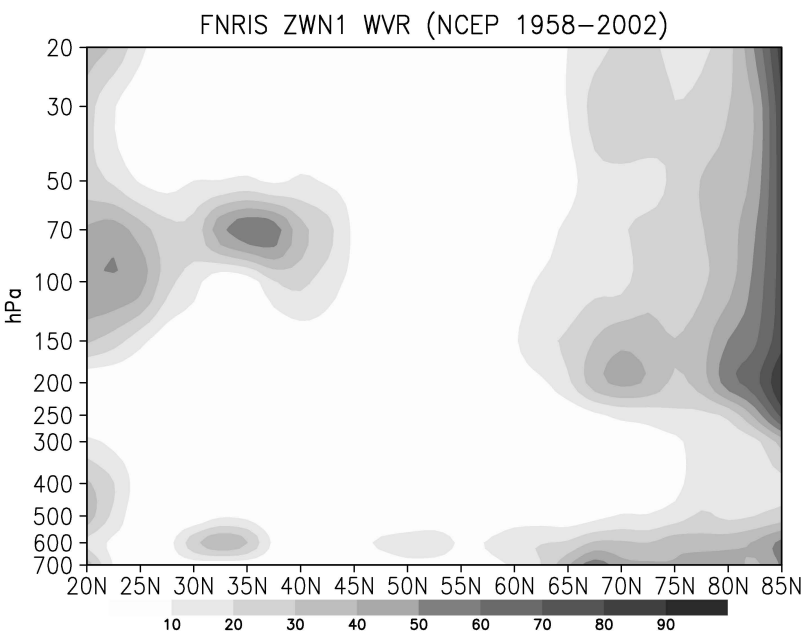

(b)
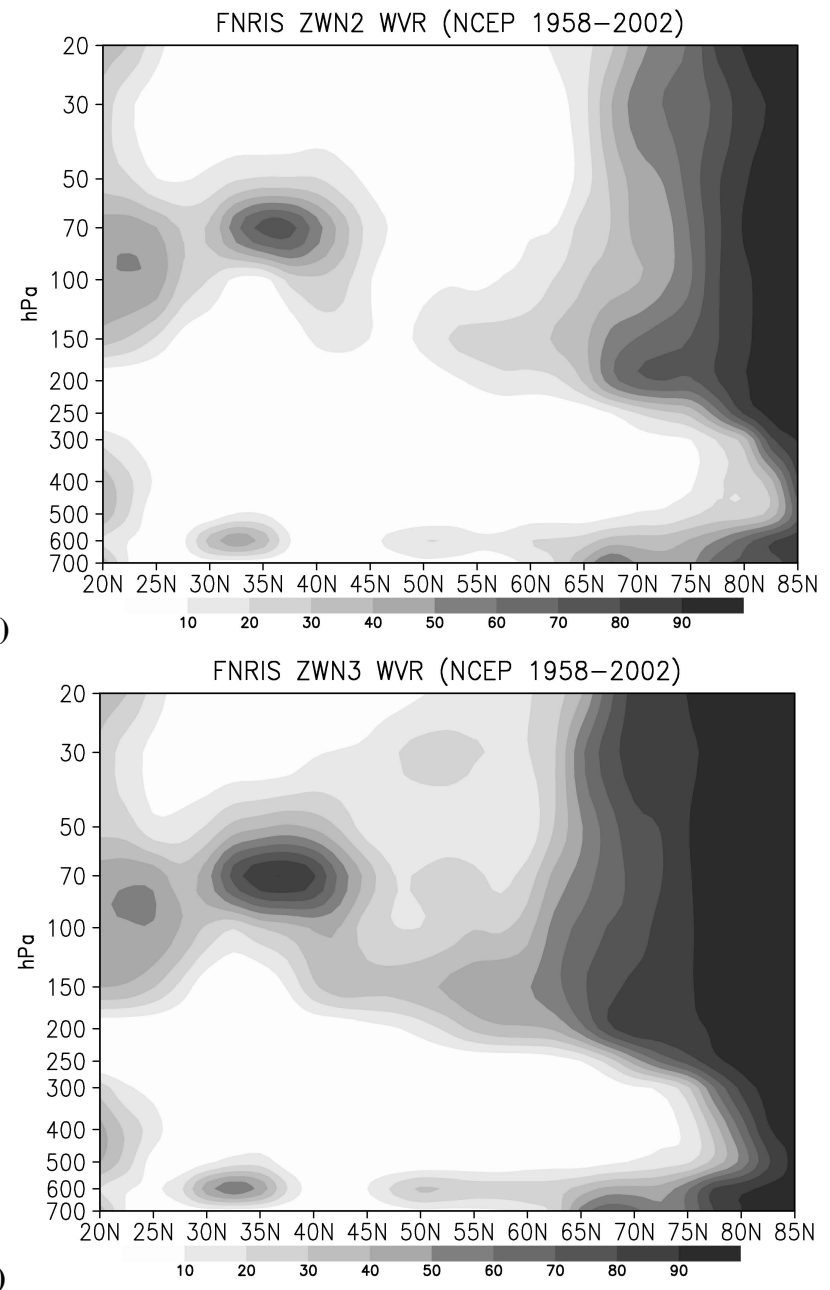

Fig. 7. $f\left(n_{k}^{2}<0\right)$ for stationary planetary waves in SVR (left column) and WVR (right column) for ZWN 1 wave (first row), ZWN 2 wave (second row) and ZWN 3 wave (bottom row). Unit of $f\left(n_{k}^{2}<0\right)$ is percent. 
(a)
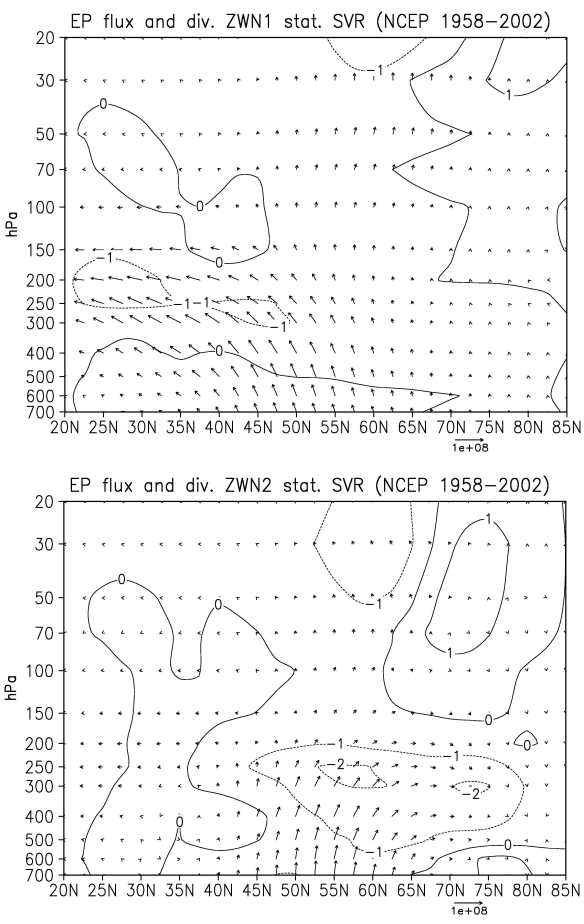

(c)

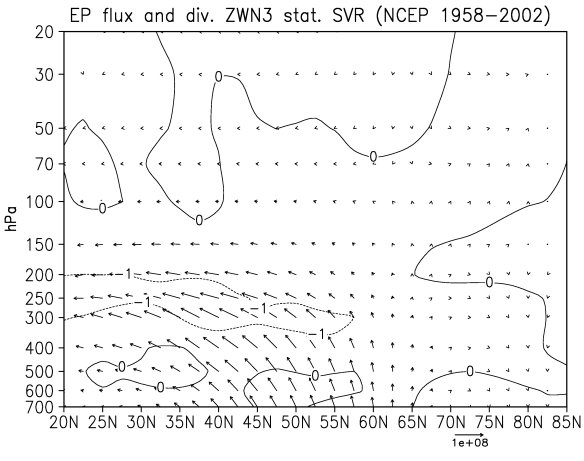

(e)

(g)

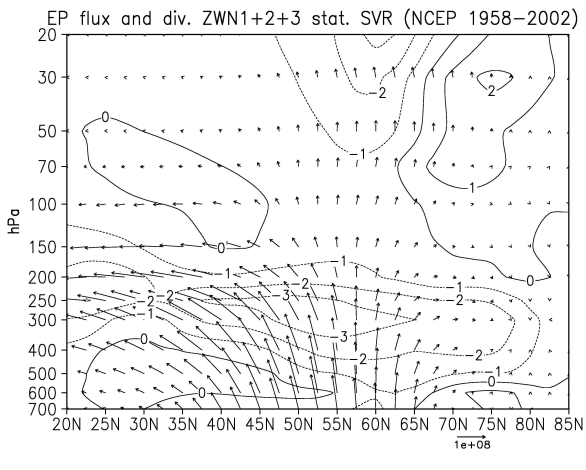

(b)
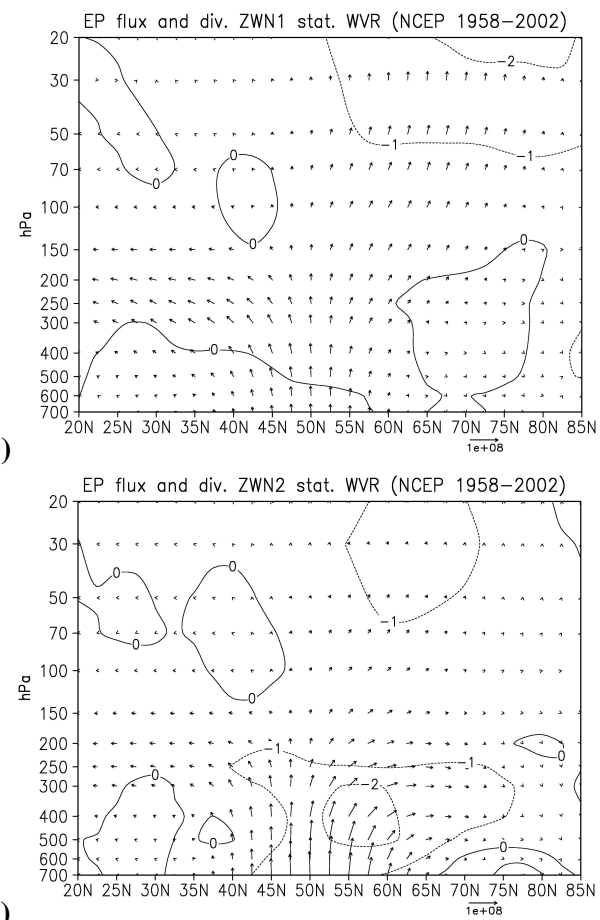

(d)

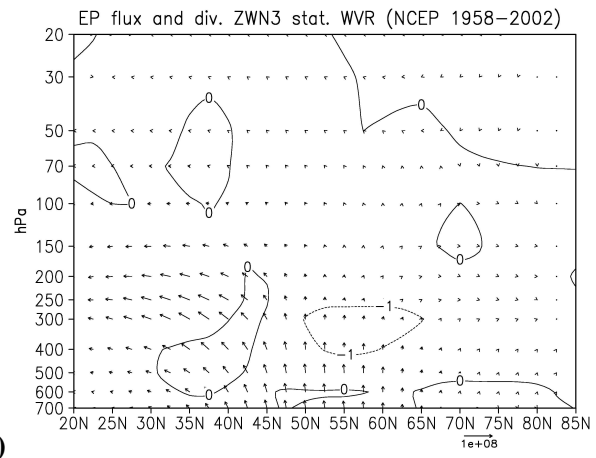

EP flux and div. ZWN1+2+3 stat. WVR (NCEP 1958-2002)

(h)

Fig. 8. Cross section of E-P flux for stationary planetary waves SVR (left column) and WVR (right column) for ZWN 1 wave (first row), ZWN 2 wave (second row), ZWN 3 wave (third row) and ZWN 1to3 waves (bottom row). Divergence contour interval is $1 \mathrm{~ms}^{-1} \mathrm{day}^{-1}$, the unit of vector is $\mathrm{kg} \mathrm{s}^{-2}$.

troposphere dominates in SVR, while in WVR the eddy heat flux into the polar stratosphere dominates. The difference between SVR and WVR of E-P flux and divergence of ZWN 1 waves (Fig. 9a) and ZWN 1+2+3 (Fig. 9d) shows similar patterns. For ZWN 3 waves, stronger equatorward vectors in the extratropical upper troposphere in SVR can be observed. 


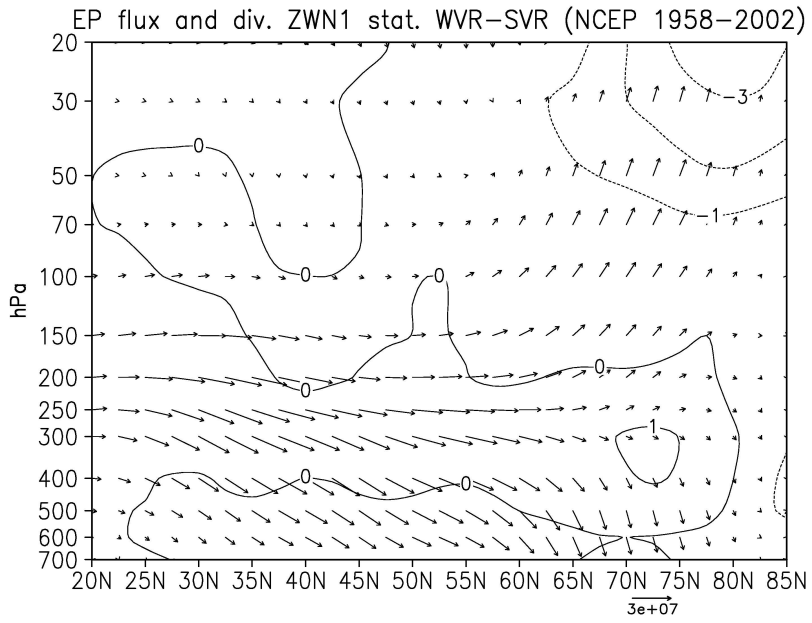

(a)

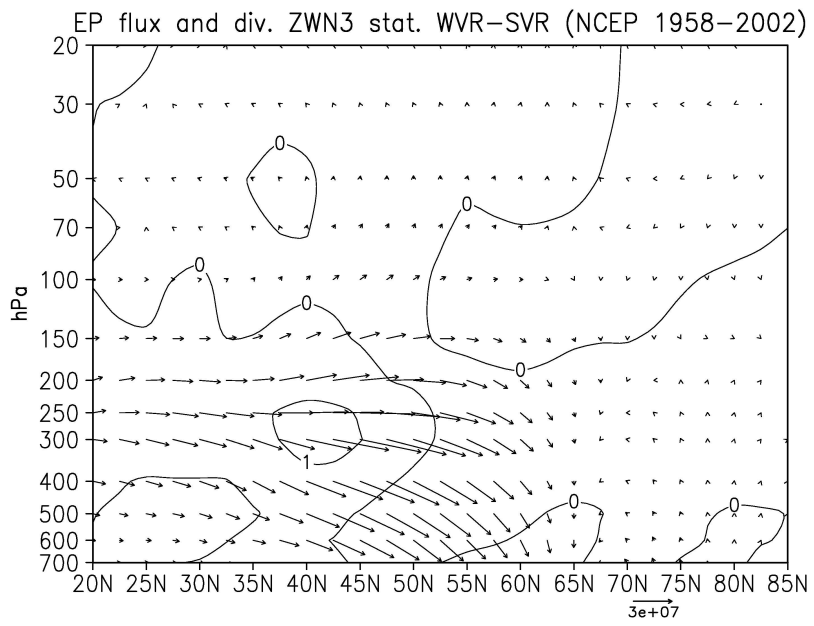

(c) (d)

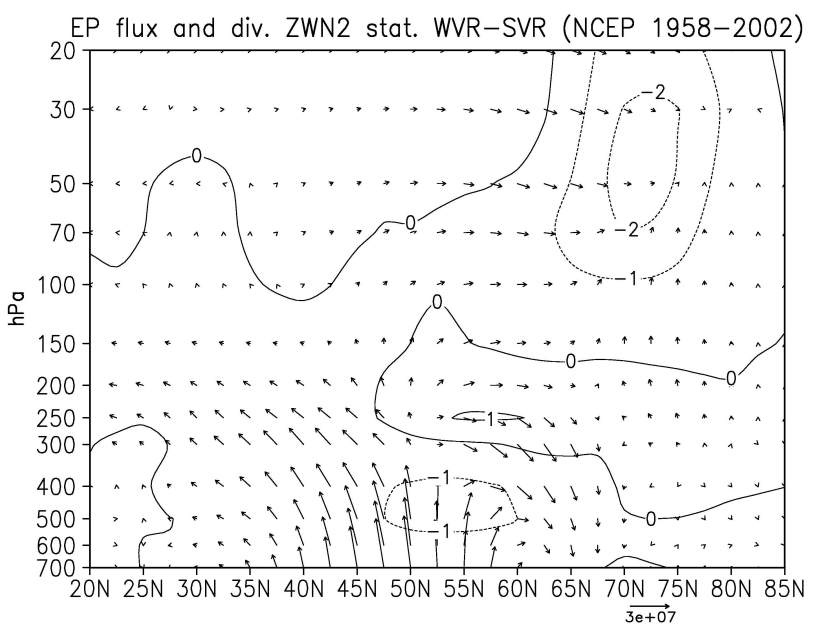

(b)

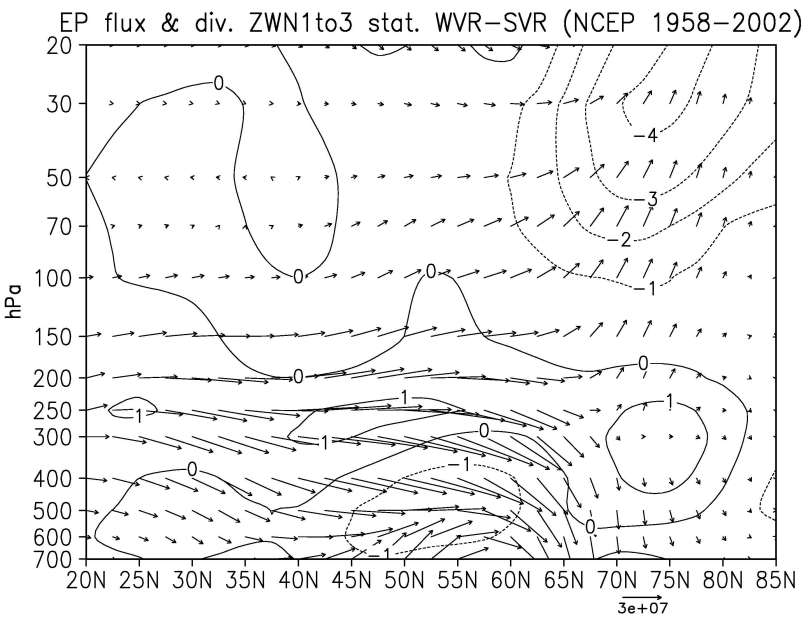

Fig. 9. Cross section of difference of E-P flux and divergence for stationary planetary waves between WVR and SVR for (a) ZWN 1 wave, (b) ZWN 2 wave, (c) ZWN 3 wave (third row), and (d) ZWN 1to3 waves. Divergence contour interval is $1 \mathrm{~ms}^{-1} \mathrm{day}^{-1}$, the unit of vector is $\mathrm{kg} \mathrm{s}^{-2}$.

However, for ZWN 2 waves, WVR shows stronger equatorward and upward vectors in the subtropical troposphere (Fig. 9b).

Finally, it should be mentioned that the positive vertical zonal wind shear is intensified in SVR (Fig. 6f) in the subpolar lower stratosphere. However, a significant influence of this positive wind shear on stationary planetary waves is not observed (Fig. 7). This is also indicated by Chen and Robinson (1992) who showed that unlike the wind shear at the subtropical tropopause, the wave propagation is not sensitive to wind shear in the stratosphere. Perlwitz and Harnik (2003) investigated the reflection of the ZWN 1 wave back to the troposphere when the polar vortex peeks at high latitudes. In this study, it is found that the ZWN 3 wave has more tendencies to be reflected back to the troposphere in any case (Figs. 2, 7).

\subsection{Correlations between E-P flux and $f\left(n_{k}^{2}<0\right)$}

In order to improve the understanding of relation between E-P flux and $f\left(n_{k}^{2}<0\right)$, and therefore the propagation of stationary planetary waves between the stratosphere and troposphere, the correlation between the vertical component of $\mathrm{E}$ $\mathrm{P}$ flux $\left(F_{z}\right.$ hereafter) and $f\left(n_{k}^{2}<0\right)$ at $70 \mathrm{hPa}, 35^{\circ}-80^{\circ} \mathrm{N}$ is analysed in Figs. 10 and 11. This analysis was performed for each DJF for all 44 winters. For single DJF, average of E-P flux was taken and $f\left(n_{k}^{2}<0\right)$ was computed for this DJF.

Figure 10 shows the scatter of $F_{z}$ and $f\left(n_{k}^{2}<0\right)$ in DJF for ZWN 1 (Fig. 10a), ZWN 2 (Fig. 10b) and ZWN 3 (Fig. 10c) waves, respectively. In Fig. 10a, the distribution shows that there is an assembly of cases with high value (up to $100000 \mathrm{~kg} \mathrm{~s}^{-2}$ ) of $F_{z}$ and small value (less than 50\%) of $f\left(n_{1}^{2}<0\right)$, which presents that the connection of strong vertical E-P flux entering the stratosphere and high chance of 
Scatter distribution between $\mathrm{Fz}$ and fnris for ZWN1 at 70hPa, 35N-80N in DJF (NCEP 19582002)
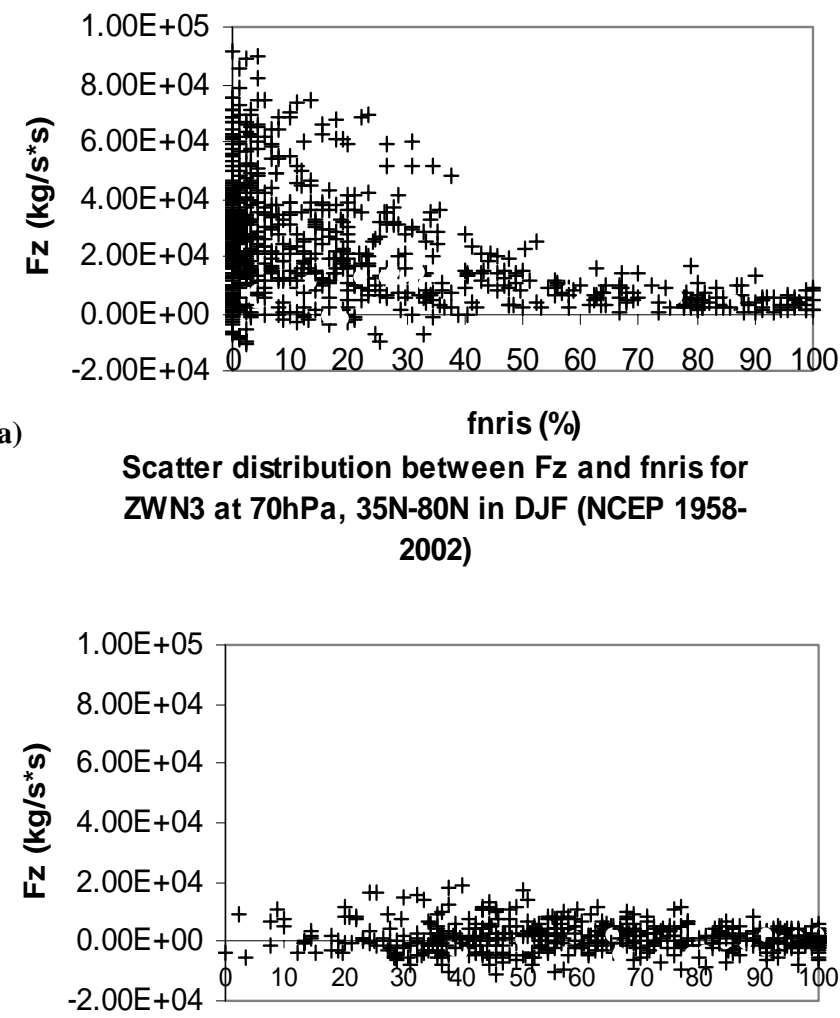

(c)

fnris (\%)
Scatter distribution between Fz and fnris for ZWN2 at 70hPa, 35N-80N in DJF (NCEP 19582002)

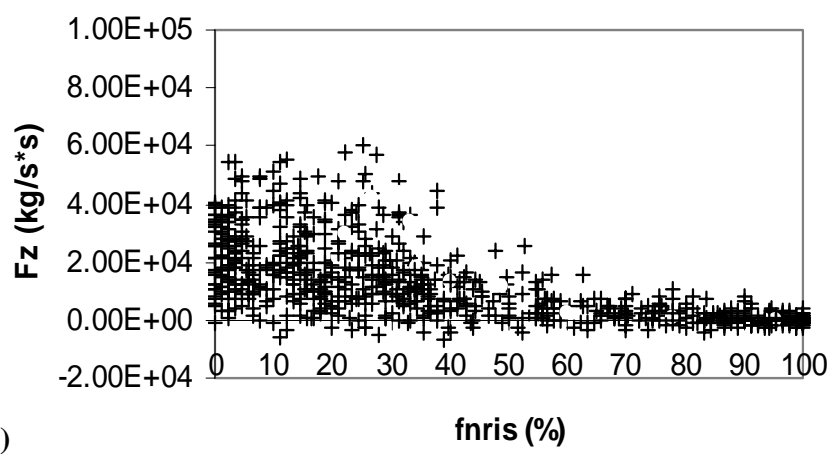

Trend lines of scatter distributions between average of $\mathrm{Fz}$ and fnris at $70 \mathrm{hPa}, 35 \mathrm{~N}-80 \mathrm{~N}$ in $\mathrm{DJF}$ (NCEP 1958-2002)

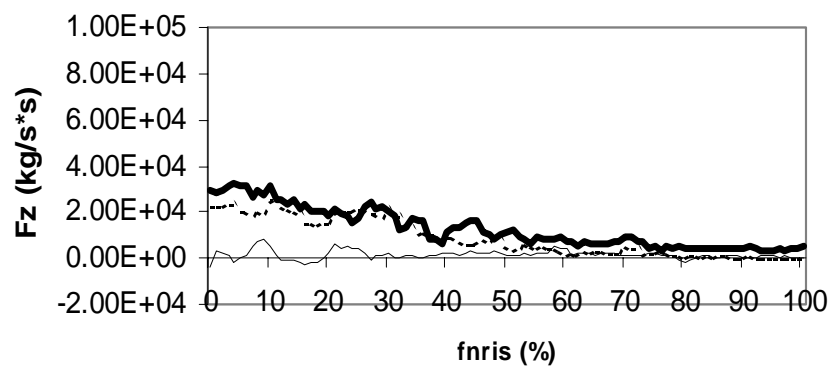

(d)

Fig. 10. Scatter distributions between vertical component of E-P flux and $f\left(n_{k}^{2}<0\right)$ at $70 \mathrm{hPa}, 35^{\circ}-80^{\circ} \mathrm{N}$ for stationary planetary waves in DJF (NCEP/NCAR RA 1958 to 2002) for ZWN 1 wave (first row), ZWN 2 wave (second row), ZWN 3 wave (third row), and the average of $F_{z}$ with $f\left(n_{k}^{2}<0\right)$ for ZWN 1,2 and 3 (bottom row).

wave propagation at $70 \mathrm{hPa}, 35^{\circ}-80^{\circ} \mathrm{N}$ for $\mathrm{ZWN} 1$ wave. For ZWN 2 wave (Fig. 10b), this assembly has a similar shape as for ZWN 1, but with smaller $F_{z}$ values (up to $60000 \mathrm{~kg} \mathrm{~s}^{-2}$ ), which indicates that although the probability of ZWN 2 wave has relatively big chance to propagate from the troposphere to the stratosphere, the realistic wave activity is not strong as ZWN 1 wave. For ZWN 3 wave (Fig. 10c), the assembly at low values of $f\left(n_{3}^{2}<0\right)$ disappeared, and most cases occur at $f\left(n_{3}^{2}<0\right)>50 \%$ having values of $F_{z} \sim 0$, which means for ZWN 3 wave, there is rare wave activity and small chance for planetary wave to propagate from the troposphere to the stratosphere. In order to clarify the difference between ZWN 1, 2 and 3 waves, the average of $F_{z}$ with fixed values of $f\left(n_{k}^{2}<0\right)$ was analysed (Fig. 10d). The average of all $F_{z}$ at each value of $f\left(n_{k}^{2}<0\right)$ was computed and 3-point running mean was performed afterwards. Figure 10d shows that, for ZWN 1 and 2 waves, when $f\left(n_{k}^{2}<0\right)<50 \%$, the values of $F_{z}$ decrease with increased values of $f\left(n_{k}^{2}<0\right)$. And the val- ues of $F_{z}$ for ZWN 1 wave are relatively bigger than ZWN 2 wave. But when $f\left(n_{k}^{2}<0\right)>50 \%, F_{z}$ are negligible for both ZWN 1 and 2 waves. For ZWN 3 wave, the values of $F_{z}$ are mostly around 0 and only small values are observed when $f\left(n_{k}^{2}<0\right)<30 \%$.

The same analysis was also performed for SVR and WVR (Fig. 11). It can be observed that the cases distribute more frequently around the area with high value of $F_{z}$ and small value of $f\left(n_{1}^{2}<0\right)$ in WVR (Figs. $11 \mathrm{~b}, \mathrm{~d}, \mathrm{f}$ ) than in SVR (Figs. 11a, c, e) for all ZWN 1, 2 and 3 waves. Therefore, it can conclude that there is higher correlation between strong wave activity and big chance of wave propagation from the troposphere to the stratosphere in WVR.

Based on this correlation analysis, the strong connection between wave activities indicated by extratropic upward E-P flux at $70 \mathrm{hPa}$ and probability of stationary planetary wave propagation is indicated. 
Scatter distribution between $\mathrm{Fz}$ and fnirs for ZWN1 at $70 \mathrm{hPa}, 35 \mathrm{~N}-80 \mathrm{~N}$ in SVR (NCEP 19582002)

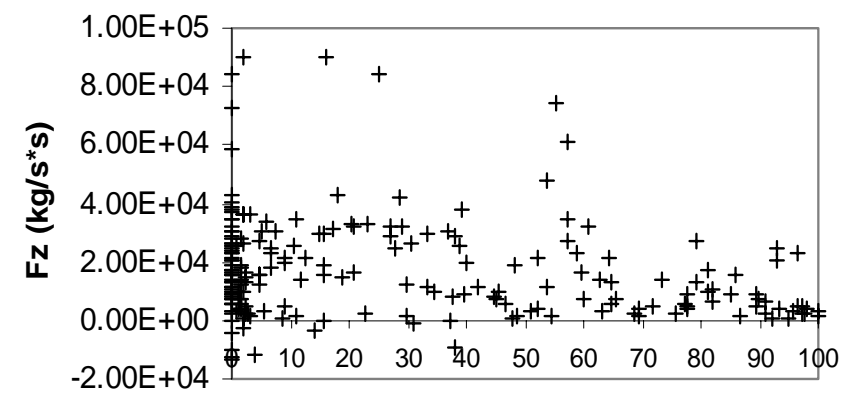

(a)

$$
\text { fnris (\%) }
$$

Scatter distribution between $\mathrm{Fz}$ and fnirs for ZWN2 at 70hPa, 35N-80N in SVR (NCEP 19582002)

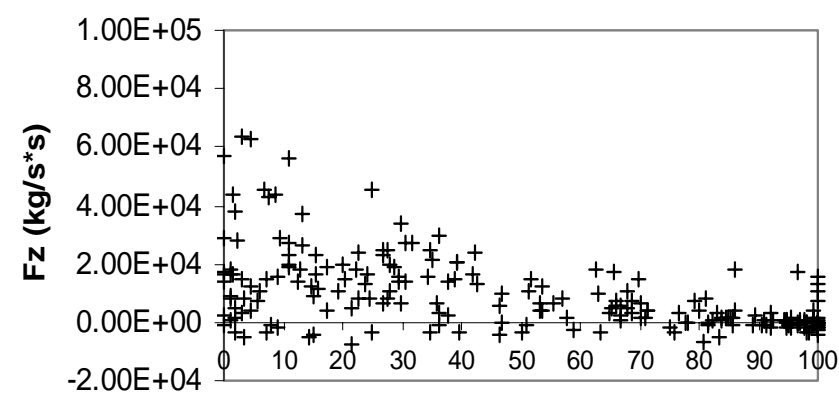

(c)

$$
\text { fnris (\%) }
$$

Scatter distribution between Fz and fnirs for ZWN3 at 70hPa, 35N-80N in SVR (NCEP 19582002)

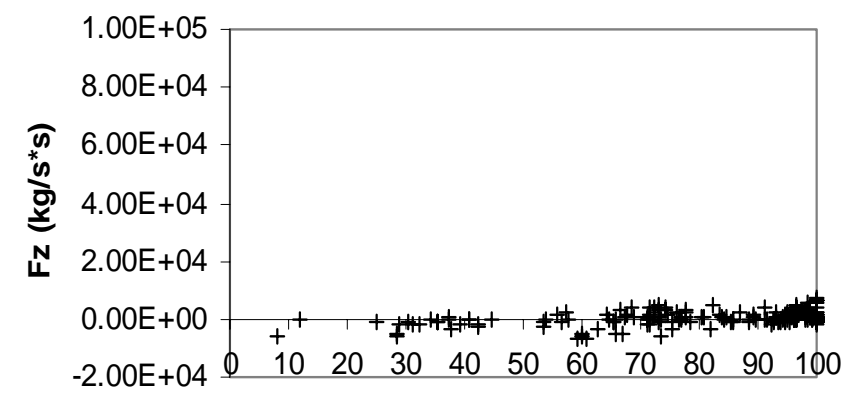

(e)

fnris (\%)

\section{Scatter distribution between Fz and fnris for ZWN1 at 70hPa, 35N-80N in WVR (NCEP 1958- \\ 2002)}

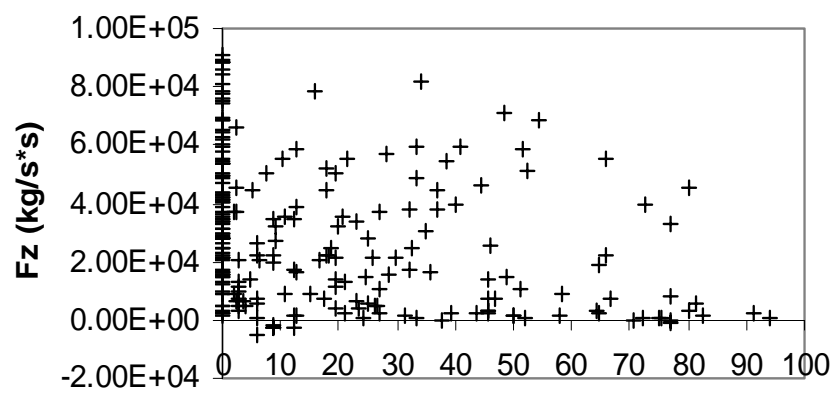

(b)

fnris (\%)

Scatter distribution between $\mathrm{Fz}$ and fnris for ZWN2 at 70hPa, 35N-80N in WVR (NCEP 1958-

2002)

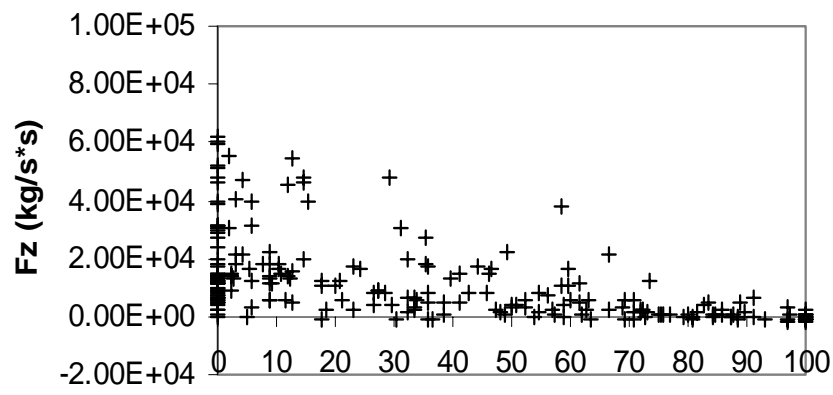

(d)

fnris (\%)

Scatter distribution between Fz and fnris for ZWN3 at 70hPa, 35N-80N in WVR (NCEP 19582002)

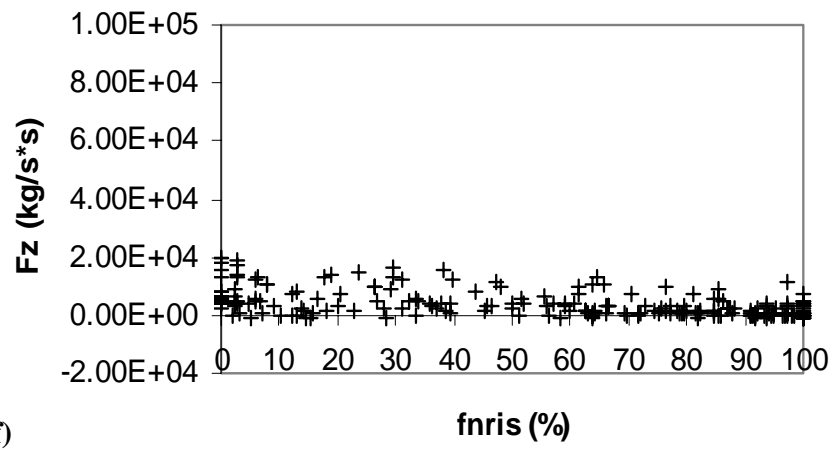

Fig. 11. Scatter distributions between vertical component of E-P flux and $f\left(n_{k}^{2}<0\right)$ at $70 \mathrm{hPa}, 35^{\circ}-80^{\circ} \mathrm{N}$ for stationary planetary waves in SVR (left column) and WVR (right column) (NCEP/NCAR RA 1958 to 2002) for ZWN 1 wave (first row), ZWN 2 wave (second row), ZWN 3 wave (third row), and the average of $F_{z}$ with $f\left(n_{k}^{2}<0\right)$ for ZWN 1, 2 and 3 (bottom row). 


\section{Summary}

The climatology of stationary planetary wave propagation in the Northern Hemisphere winter is analyzed based on the NCEP-NCAR reanalysis data 1958-2002. The frequency of negative refractive index squared $f\left(n_{k}^{2}<0\right)$ is introduced and applied to study the potential influence of the atmospheric mean flow on wave propagation. This zonal wind-dependent refractive index shows the control of atmospheric zonal flow on planetary wave propagation. As observed and published elsewhere, not only the zonal mean zonal wind but also the vertical shear of zonal wind affects the possibility of stationary planetary wave propagation. The zonal mean flow controls the propagation based on the ZWN-depended critical Rossby velocity (Charney and Drazin, 1961). The wind shear above the subtropical tropopause also influences planetary wave propagation (Chen and Robinson, 1992; Hu and Tung, 2002). The sensitivity of wave propagation to atmospheric stability is also found by studying the influence of buoyancy frequency variations on $f\left(n_{k}^{2}<0\right)$. It is found that the atmospheric stability can also partly control the stationary planetary wave propagation, especially around the extratropical tropopause area. On the other hand, the E-P flux and divergence (Andrews et al., 1987) illustrates the eddy forcing on the mean flow. In Northern Hemisphere winter, stationary planetary waves have a big chance to propagate from the troposphere to the stratosphere for ZWN 1 and ZWN 2 waves. This also corresponds with the branch of upward E-P fluxes on the distribution of cross section of E-P flux (Figs. 5, 8). To summarize, planetary wave propagation is controlled not only by distribution of atmospheric zonal wind and wind shear, but also by distribution of atmospheric stability. The variation of atmospheric stability with latitudes is considerable. On the other hand, the planetary waves exert eddy momentum flux in the subtropical troposphere and eddy heat flux in the subpolar stratosphere. Waves also induce eddy momentum forcing on the atmospheric mean flow in both the troposphere and the stratosphere at middle latitudes.

The correlation between wave activity and probability of wave propagation was also analysed at in this paper. And the strong connection between the stationary planetary wave activity and probability of propagation from the troposphere to the stratosphere was observed. Strong E-P fluxes to the NH stratosphere occur when and where the frequency of negative refractive index squared is smaller than $50 \%$, for ZWN 1 and 2. ZWN 3 wave related E-P fluxes are not effective, even in the rare cases of low frequency of negative refractive indices squared.

The anomalies of mean flow in Northern Hemisphere winter were isolated into two major regimes - strong polar vortex (SVR) and weak polar vortex (WVR) considering time series of the zonal wind at $50 \mathrm{hPa}$ and $65^{\circ} \mathrm{N}\left(\bar{u}_{50}\left(65^{\circ} \mathrm{N}\right)\right.$ as a diagnostic. It is found that the SVR lasts longer than WVR. Variations of the polar vortex regimes can be related to the different propagation structures of planetary waves (Limpasu- van and Hartmann, 2000; Perlwitz and Graf, 2000a; Walter, 2003). The analysis of $f\left(n_{k}^{2}<0\right)$ shows that the anomalies of stratospheric circulation influence the probability of stationary planetary wave propagation. In WVR, planetary waves have more possibility to propagate from the troposphere to the stratosphere than in SVR. This possibility decreases with increasing ZWNs. Generally, planetary waves show stronger propagation in WVR from the troposphere to the stratosphere. In SVR, the planetary waves lead to strong westerly forcing in polar stratosphere while in WVR the easterly forcing prevails in both polar and sub-polar stratosphere. For planetary wave activity, the equatorward eddy momentum flux in the extratropical upper troposphere is much stronger in SVR, while the eddy heat flux from troposphere upward into the stratosphere around northern pole is much stronger in WVR. In general, the probability of negative refractive index squared proves as a relatively simple but nevertheless powerful tool to investigate planetary wave propagation conditions. It allows to also considering other than mean wind and may be applied to assess atmospheric models' performance in planetary wave propagation.

Acknowledgements. This work was supported by German BMBF within the AFO2000 project.

Edited by: M. Dameris

\section{References}

Andrews, D. G.: Wave, mean-flow interaction in the middle atmosphere, Adv. Geophys., 28A, 249-275, 1985.

Andrews, D. G., Holton, J. R., and Leovy, C. B.: Middle Atmosphere Dynamics, Academic Press Inc., 489 pp, 1987.

Baldwin, M. P. and Dunkerton, T. J.: Propagation of the Arctic Oscillation from the stratosphere to the troposphere, J. Geophys. Res., 104, 30 937-30 946, 1999.

Baldwin, M. P. and Dunkerton, T. J.: Stratospheric harbingers of anomalous weather regimes, Science, 294, 581-584, 2001.

Boville, B. A.: The influence of the polar night jet on the tropospheric circulation in a GCM, J. Atmos. Sci., 41, 1132-1142, 1984.

Castanheira, J. M. and Graf, H.-F.: North Pacific-North Atlantic relationships under stratospheric control?, J. Geophys. Res., 108, 4036, doi:10.1029/2002JD002754, 2003.

Charney, J. G. and Drazin, P. G.: Propagation of planetary-scale disturbances from the lower into the upper atmosphere, J. Geophys. Res., 66, 83-109, 1961.

Chen, P. and Robinson, W. A.: Propagation of planetary waves between the troposphere and stratosphere, J. Atmos. Sci., 49, 25332345, 1992.

Chen, W., Graf, H.-F., and Takahashi, M.: Observed interannual oscillation of planetary wave forcing in the Northern Hemisphere winter, Geophys. Res. Lett., 29, 2037, doi:10.1029/2002G1016062, 2002.

Christiansen, B.: Evidence of nonlinear climate change: Two stratospheric regimes and a regime shift, J. Climate, 16, 3681-3690, 2003. 
Dickinson, R. E.: Planetary Rossby waves propagating vertically through weak westerly wind wave guides, J. Atmos. Sci., 25, 984-1002, 1968.

Eliassen, A. and Palm, E.: On the transfer of energy in stationary mountain waves, Geofys. Publ., 22-3, 1-23, 1961.

Gillett, N. P., Allen, M. R., Mcdonald, R. E., Senior, C. A., Shindell, D. T., and Schmidt, G. A.: How linear is the Arctic Oscillation response to greenhouse gases?, J. Geophys. Res., 107, D3, doi:10.1029/2001JD000589, 2002b.

Hartmann, D. L. and Lo, F.: Wave-driven zonal flow vacillation in the Southern Hemisphere, J. Atmos. Sci., 55, 1303-1315, 1998.

$\mathrm{Hu}, \mathrm{Y}$. and Tung, K. K.: Interannual and decadal variations of planetary wave activity, stratospheric cooling, and northern hemisphere annular mode, J. Climate, 15, 1659-1673, 2002.

Huang, R. and Gambo, K.: The response of a Hemispheric MultiLevel Model Atmosphere to Forcing by Topography and Stationary Heat Sources (I) Foring by Topography, J. Meteorol. Soc. Japan, 60, 78-92, 1982.

Kalnay, E., Kanamitsu, M., Kistler, R., Collins, W., Deaven, D., Gandin, L., Iredell, M., Saha, S., White, G., Woollen, J., Zhu, Y., Chelliah, M., Ebisuzaki, W., Higgins, W., Janowiak, J., Mo, K. C., Ropelewski, C., Wang, J., Leetmaa, A., Reynolds, R., Jenne, R., and Joseph, D.: The NCEP/NCAR 40-year reanalysis project, Bull. Amer. Meteor. Soc., 77, 437-472, 1996.

Kistler, R., Kalnay, E., Collins, W., Saha, S., White, G., Woollen, J., Chelliah, M., Ebisuzaki, W., Kanamitsu, M., Kousky, V., van den Dool, H., Jenne, R., and Fiorino, M.: The NCEP-NCAR 50-year reanalysis: Monthly means CD-ROM and documentation, Bull. Amer. Meteorol. Soc., 82, 247-268, 2001.

Limpasuvan, V. and Hartmann, D. L.: Wave-maintained annular modes of climate variability, J. Climate, 13, 4414-4429, 2000.

Limpasuvan, V., Hartmann, D. L., Thompson, D. W. J., Jeev, K., and Yung, Y. L.: Stratosphere-Troposphere evolution during polar vortex intensification, J. Geophys. Res., 110, D24101, doi:10.1029/2005JD006302, 2005.

Lin, B.: The behavior of winter stationary planetary waves forced by topagraphy and diabatic heating, J. Atmos. Sci., 39, 12061226, 1982.
Matsuno, T.: Vertical propagation of stationary planetary waves in the winter Northern Hemisphere, J. Atmos. Sci., 27, 871-883, 1970.

Mukougawa, H. and Hirooka, T.: Predictability of stratospheric sudden warming: A case study for 1998/99 winter, Mon. Wea. Rev., 132, 1764-1776, 2004.

Palmer, T. N.: A nonlinear dynamical perspective on climate change, Weather, 48, 314-326, 1993.

Perlwitz, J. and Graf, H.-F.: The statistical connection between tropospheric and stratospheric circulation of the Northern Hemisphere in winter, J. Climate, 8, 2281-2295, 1995.

Perlwitz, J.: The Dynamical Link Between the Troposphere and Stratosphere and its Potential to Affect Climate, $\mathrm{Ph}$. D. dissertation, Max-Planck-Institut fuer Meteorologie, Hamburg, 145 pp, 2000.

Perlwitz, J. and Graf, H.-F.: Troposphere-stratosphere dynamic coupling under strong and weak polar vortex conditions, Geophys. Res. Lett., 28, 271-274, 2001a.

Perlwitz, J. and Graf, H.-F.: The variability of the horizontal circulation in the troposphere and stratosphere - a comparison, Theor. Appl. Climatol., 69, 149-161, 2001b.

Perlwitz, J. and Harnik, N.: Observational evidence of a stratospheric influence on the troposphere by planetary wave reflection, J. Climate, 16, 3011-3026, 2003.

Schoeberl, M. R. and Geller, M. A.: A calculation of the structure of stationary planetary waves in winter, J. Atmos. Sci., 34, 1235$1255,1977$.

Walter, K.: Changing patterns of tropospheric variability in the north Atlantic region, Ph.D dissertation, Max Planck Institute for Meteorology, 157 pp, 2003.

Walter, K. and Graf, H.-F.: The North Atlantic variability structure, storm tracks, and precipitation depending on the polar vortex strength, Atmos. Chem. Phys., 5, 239-248, 2005, http://www.atmos-chem-phys.net/5/239/2005/. 